Mittheilung aus dem chemischen Laboratorium der Akademie der Wissenschaften zu München.

\author{
Ueber Diamidophenylosotriazol; \\ von Johannes Thiele und Karl Schleussnor.
}

\title{
Theoretischer Theil.
}

Der Stickwasserstoff $\mathrm{NH}_{2} \cdot \mathrm{NHI}_{\mathrm{NH}}$ (Prozan), das höhere Homologe des Hydrazins, oder Alkylderivate desselben sind bisher nicht erhalten worden, da es bis jetzt nicht gelungen ist, Nitrosohydrazine oder Diazoamidoverbindungen ohne Sprengung der Stickstoffbindung zu reduciren. Einige Beobachtungen über amidirte Azole, welche im hiesigen Laboratorium gemacht wurden, schienen nun einen Weg zur Darstellung von Prozanderivaten anzudeuten. Thiele und Ingle ${ }^{1}$ ), sowie Manchot ${ }^{2}$ ) fanden nämlich, dass das Amidotetrazol und amidirte Triazole durch Säuren weit leichter sprengbar seien, als die nicht amidirten Ringe. Wenn sich diese .Reaction auf amidirte Osotriazole, etwa auf einen Körper

$$
\mathrm{C}_{6} \mathrm{H}_{5}-\mathrm{N} \sum_{\mathrm{N}=\mathrm{C}-\mathrm{NH}_{2}}^{\mathrm{N=C}-\mathrm{NH}_{3}}
$$

übertragen liess, war als Sprengstück das Phenylprozan

20 erwarten.$$
\mathrm{C}_{6} \mathrm{H}_{5}-\mathrm{N}<\mathrm{NH}_{2}
$$

Amidirte Osotriazole waren zur Zeit als vorliegende Arbeit begonnen wurde, noch nicht bekannt. Zur Darstellung dieses Diamidophenylosotriazols stellt man zunächst durch Addition von Hydroxylamin an Dicyanphenylhydrazin das Oxalenphenylhydrazidamidoxim dar ${ }^{3}$ ):

1) Diese Annalen 287, 234.

2) Dissertation, München 1895 .

3) Dieses Amidoxim ist schon vor längerer Zeit im hiesigen Iaboratorium von Herrn Dr. Stang.e dargestellt worden, der auch einige Annalen der Chemie 295. Bd. 


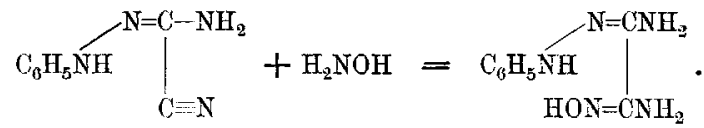

Durch Erhitzen mit Wasser unter Druck lässt sich dieser Körper leicht in das gesuchte Diamin umwandeln:

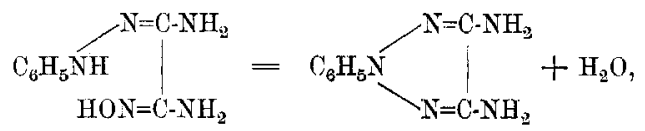

indessen war die gewünschte Spaltung bis jetzt nicht ausfülrbar. Beim Erhitzen mit Salzsäure auf hohe Temperaturen entstand im Wesentlichen ein amorpher, blauschwarzer, unschmelzbarer Körper, neben Ammoniak, Stickstoff und Kohlensäure, ebenso bewirkten Kalilauge oder Sodalösung bei hohen Temperaturen nur die Bildung hochmolekularer Condensationsproducte. Ebensowenig gelang bisher die Hydrirung des Osotriazolringes, die möglicherweise $\mathrm{zu}$ einem leichter sprengbaren Körper geführt hätte. Dic Versuche in beiden Richtungen sollen jedoch fortgesetzt werden. Trotz dieser für den eigentlichen Zweck der Arbeit negativen Resultate hat das Diamidophenylosotriazol indess in anderer Hinsicht einige interessante Reactionen gezeigt.

Das Diamidophenylosotriazol ist eine schwache, nur einsäurige Base, deren Salze leicht dissociiren; mit Silbernitrat bildet es eine schwer lösliche Verbindung zu gleichen Molekülen.

Bei der Oxydation mit Fisenchlorid entsteht ein amorpher, blaner, azinartiger Farbstoff, wahrscheinlich von der Formel

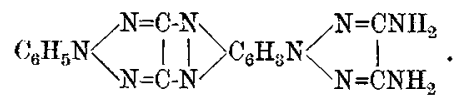

Seine Constitution ergiebt sich mit grosser Wahrscheilllichkeit aus dem analogen Verhalten anderer Orthodiamine und aus der Zusammensetzung der schön krystallisirenden Leukoverbindung. Dass bei der Entstehung dieses Farbstoffes die

Condensationsversuche damit ansgeführt hat. Da der Körper bisher noch nicht beschrieben ist, so ist seine Darstellung und die eines auch schon von Horrn Dr. Stange dargestellten Acetates in den folgenden experimentellen Theil aufgenommen worden. 
Amidogruppen des Osotriazolringes in den Benzolkern eines zweiten Moleküls des Diamins eingegriffen haben, goht daraus hervor, dass am Stickstofi substituirtes Diamidophenylosotriazol, ebenso wie Diamidophenylosotriazolsulfosäure mit Eisenchlorid keine analogen Farbstoffe liefern.

Mit Orthodiketonen bildet das Diamin Chinoxalinderivate. Die Condensation erfolgt leicht und ist für die Orthostellung der beiden Amidogruppen beweisend. Sie verläuft mit Benzil und Phenanthrenchinon im Sinne der Formeln :

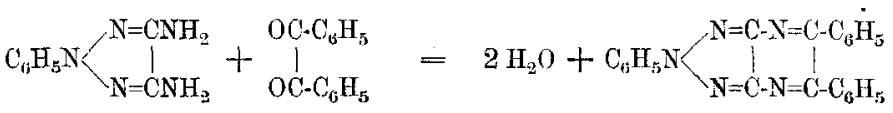

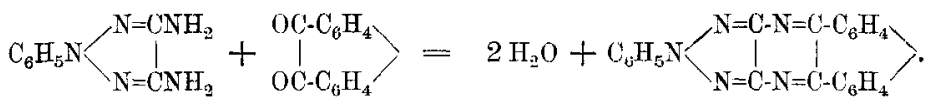

Weit schwieriger erfolgt die Anlagerung eines fünfgliedrigen Ringsystemes an den Osotriazolring. Während Orthodiamine des Benzols und seiner Homologen äusserst leicht Imidazole oder Azimide bilden, konnte erstere Reaction bei dem Diamidosotriazol gar nicht, letztere nur unter besonderen Bedingungen ausgeführt werden.

So entsteht durch Einwirkung von Benzaldehyd keine Anhydrobase, sondern eine wahre Benzylidenverbindung. Dass dem betreffenden Condensationsproducte die Formel eines Dibenzaldiamidophenylosotriazols:

$$
\mathrm{C}_{6} \mathrm{H}_{5} \mathrm{~N}\left\langle\begin{array}{l}
\mathrm{N}=\mathrm{C} \cdot \mathrm{N}=\mathrm{CH} . \mathrm{C}_{6} \mathrm{H}_{5} \\
\mathrm{~N}=\mathrm{C} \cdot \mathrm{N}=\mathrm{C} \amalg . \mathrm{C}_{6} \mathrm{H}_{5}
\end{array}\right.
$$

zukommt, geht aus seinem Verhalten gegen verdünnte Säuren zweifellos hervor. Dieselben spalten schon in der Kälte, leicht beim Erhitzen, Benzaldehyd ab. Das aus IDiamidophenylosotriazol und Salicylaldehyd erhaltene Dioxybenzaldiamidophenylosotriazol verhält sich ganz analog.

In ähnlicher Weise bildet sich beim Kochen des Diamins mit Eisessig kein Imidazol, sondern Monoacetyldiamidophenylosotriazol, neben Diacetyldiamidophenylosotriazol. Durch Essig- 
säureanhydrid oder Acetylchlorid werden selbstverständlich beide Amidogruppen acetylirt, mit Benzoylchlorid entsteht Dibenzoyldiamidophenylosotriazol.

Ob unter geeigneten Bedingungen auch die Imidazole dargestellt werden können, muss dahin gestellt bleiben. Die in dieser Richtung gemachten Versuche ergaben negative Resultate. Sicher erfolgt die Ringschliessung nur schwierig.

Lässt man unter geeigneten Umständen salpetrige Säure auf Diamidophenylosotriazol einwirken, so entsteht eine einfache Diazoverbindung. Dieselbe wurde als Pikrat und als Dimethylanilinazoamidophenylosotriazol isolirt. Ist ihre Entstehung an und für sich schon merkwürdig, so darf ihr aussergewöhnliches Verhalten ein noch grösseres Interesse beanspruchen. Ihre Salze sind nicht explosiv. Lösungen in verdünnten Mineralsäuren kuppeln selbst nach längerem Kochen noch mit Aminen und Phenolen. Sie verlieren diese Fähigkeit bei gewöhnlicher Temperatur nur langsam - nach sechs bis acht Tagen rascher bei Zusatz von Natriumacetat. Das Amidodiazophenylosotriazol geht dabei unter Abspaltung von Wasser in sein inneres Anhydrid, das Phenylosotriazolazimid, über:

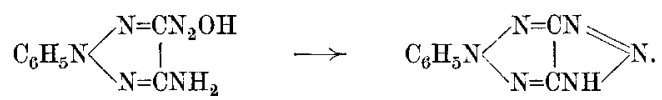

Das intermediäre Auftreten einer Diazoverbindung bei der Azimidbildung, das bekanntlich bisher noch nicht beobachtet worden ist, steht im Einklange mit der unsymmetrischen Formel und der Auffassung der Azimide als ringförmige Diazoamidoverbindungen. Während aber die bekannten Azimide in ihren Eigenschaften von den Diazoamidoverbindungen total abweichen und besonders durch eine ausserordentliche Beständigkeit ausgezeichnet sind, zeigt das Phenylosotriazolazimid in vieler Hinsicht das charakteristische Verhalten der Diazoamidokörper.

Starke Säuren sprengen, besonders beim Erwärmen, den Azimidring unter Rückbildung des Diazosalzes. Daher gelingt es auch nicht, das Azimid durch Acetylchlorid zu acetyliren, sondern man erhält glatt Diazoacetamidophenylosotriazolchlorid: 


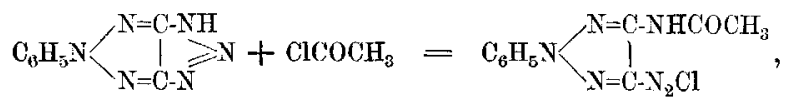

das man in Lösung auch durch Diazotiren des Acetyldiamidophenylosotriazols erhalten kann. Dieses Diazochlorid zeigt in ganz besonderem Grade die Eigenthümlichkeit der Diazoverbindungen der Azole, leicht in die Isoform überzugehen, offenbar infolge der starken Anhäufung negativer Gruppen. Es kuppelt zwar beim Eingiessen seiner Lösung in eine Sodalösung von $\mathrm{R}$-Salz, mit einer auch nur mässig ätzalkalischen $\mathrm{R}$-SalzLösung tritt dagegen keine Farbstofibildung ein, offenbar weil die Bildung der Isoform hier rascher erfolgt, wie die Kuppelung.

Behandelt man das Diazoacetamidophenylosotriazolchlorid mit Natriumacetat, so scheidet sich das Phenylosotriazolacetylazimid aus:

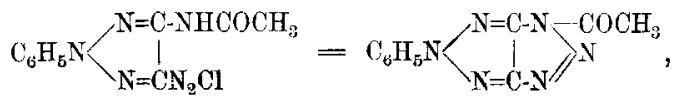

welches durch Alkalien leicht in das nicht acetylirte Azimid verwandelt wird. Das Acetylazimid zeichnet sich ganz besonders durch leichte Sprengbarkeit des Azimidringes aus. Es kuppelt, gerade wie eine Diazoamidoverbindung, in Eisessiglösung schon in der Kälte mit Dimethylanilin, ja beim Kochen mit Alkohol geht es unter Entweichen von Stickgas und Aldehyd sogar in Acetylamidophenylosotriazol über, offenbar, indem vorher Acetylamidodiazophenylosotriazol sich bildet, welches auch direct in Acetylamidophenylosotriazol verwandelt werden kann:

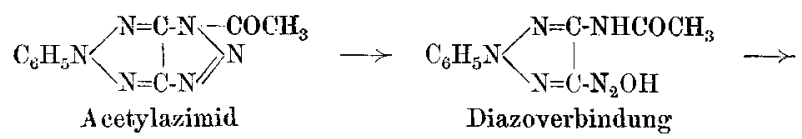

$$
\mathrm{C}_{4} \mathrm{H}_{5} \mathrm{~N} / \mathrm{N}=\mathrm{C}-\mathrm{NHCOCH}_{3}
$$

Acetylamidophenylosotriazol.

Durch Abspaltung des Acetyls aus dem Acetylamidophenylosotriazol wurde Amidophenylosotriazol 
134 Thiele u. Schleussner, Diamidophenylosotriazol.

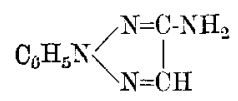

erhalten, welches wie die anderen amidirten Azole das Verhalten eines primären aromatischen Amins zeigt.

Oxyamidophenylosotriazol

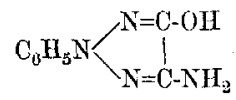

entsteht in geringer Menge als Nebenproduct bei der Bildung des Diamidophenylosotriazols, vielleicht aus einer intermediär gebildeten Hydroxamsäure,

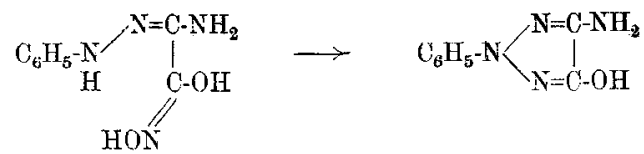

da der fertige Diamidokörper kein Amid gegen Hydroxyl auszutauschen scheint. Der Körper ist eine schwache Säure, mit Benzaldehyd liefert er ein Benzylidenderivat, mit salpetriger Säure eine kuppelungsfähige Diazoverbindung.

Während dieser Versuche wurde gefunden ${ }^{4}$ ), dass das Phenylamidotriazol

$$
\underset{\mathrm{N}=\mathrm{C}}{\mathrm{C}_{6} \mathrm{H}_{5}-\mathrm{C}=\mathrm{N}} \underset{\mathrm{NH}_{2}}{\mathrm{NH}}
$$

im Gegensatze zu dem Amidotriazol durch Salzsäure kaum mehr spaltbar sei. Es erschien daher nicht unmöglich, dass ein Diamidoosotriazol<smiles></smiles>

ebenfalls sich im Gegensatze zum Phenylderivat als spaltbar erwiese. Es wurden daher noch einige Versuche angestellt, einen solchen Körper aus Amidoguanidin oder Semicarbazid ähnlich wie das Diamidophenylosotriazol aus Phenylhydrazin darzustellen.

4) Benack, Inaug.-Dissert. München 1896. 
Amidoguanidin erwies sich indessen für vorliegenden Zweck wegen der Zersetzlichkeit der freien Base als ungeeignet. Es reagirt zwar mit Cyan, indem eine Base entsteht, deren Nitrat die Formel $\mathrm{C}_{4} \mathrm{~N}_{10} \mathrm{H}_{12} \mathrm{O}_{8}$ hat. Dasselbe ist vielleicht das Nitrat des noch nicht bekannten, dem Cyanphenylhydrazin entsprechenden Cyansemicarbazids:

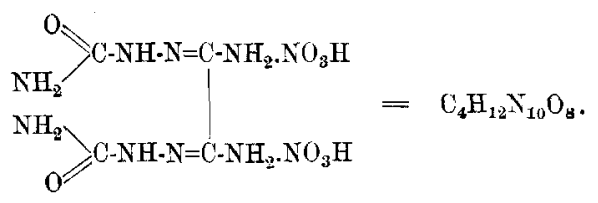

In Bezug auf den eigentlichen Zweck der vorliegenden Arbeit war dieses Additionsproduct ohne Interesse und ist in Folge dessen nicht näher untersucht worden.

Semicarbazid addirt hingegen ein Molekül Dicyan unter Bildung einer Verbindung, welche nach ihrer Bildungsweise als Dicyansemicarbazid,<smiles>NC(=O)N=C(N)N</smiles>

zu bezeichnen ist. Dieses geht mit Hydroxylamin quantitatir in Dicyansemicarbazidamidoxim,<smiles>NC(=O)N=C(N)NC(N)=O</smiles>

uber. Durch Erhitzen mit Wasser liefert letzteres zwei Verbindungen von den Formeln $\mathrm{C}_{3} \mathrm{~N}_{5} \mathrm{H}_{5} \mathrm{O}_{2}$ und $\mathrm{C}_{6} \mathrm{~N}_{8} \mathrm{H}_{14} \mathrm{O}_{7}$. Dic Constitutionsformeln beider Verbindungen bedürfen noch näherer Feststellung, jedenfalls liefern sie bei der Spaltung kein Prozan, sondern Hydrazin. Die Verbindung $\mathrm{C}_{3} \mathrm{~N}_{5} \mathrm{H}_{5} \mathrm{O}_{2}$ könnte entstanden sein nach der Gleichung:

$$
\mathrm{C}_{3} \mathrm{~N}_{6} \mathrm{H}_{8} \mathrm{C}_{2}=\mathrm{C}_{3} \mathrm{H}_{5} \mathrm{~N}_{5} \mathrm{~N}_{2}+\mathrm{NH}_{3}
$$

Dicyansemi-

carbazidamidoxim.

Schliesslich wurden noch Versuche angestellt, um ein $D i$ oxyphenylosotriazol oder Oxalylphenylprozan, 
136 Thiele u. Schleussner, Diamidophenylosotriazol.

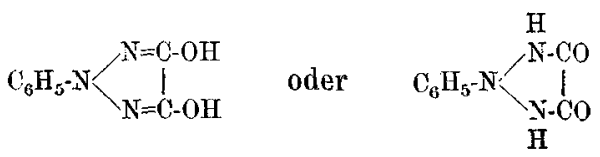

darzustellen.

Phenylhydrazidoxalester (Oxalsäuremonophenylhydrazidäthylester ${ }^{\tilde{j}}$ ) reagirt mit Hydroxylamin unter Bildung von Phenylhydrazidoxalhydroxamsäure,

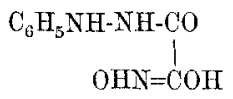

Dieselbe giebt mit Essigsäureanhydrid leicht ein Acetylderivat von der Formel

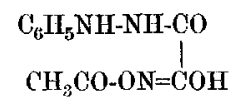

Beim Erhitzen mit Dimethylanilin spaltet dieses leicht Essigsäure $a b$, und man sollte das Oxalylphenylprozan erwarten,

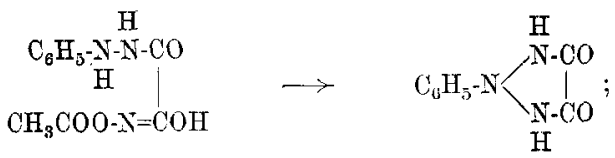

statt dessen entsteht aber in Folge einer Umlagerung, die eine gewisse Aehnlichkeit mit der Beckmann'schen Umlagerung besitzt und vor allem mit der von Lossen beschricbenen Bildung von Diphenylharnstoff aus Dibenzhydroxamsäure, das schon von Pinner ${ }^{6}$ ) beschriebene Phenylurazol,<smiles>[R]OC(=O)NC(=O)NCCCC</smiles>

5) Bülow, diese Amalen $\mathbf{2 3 6}, 197$.

6) Ber. d. dentsch. chem. Ges. 20, 2360; 26, 1219. 


\section{Experimenteller Theil.}

\section{Derivate des Phenylosotriazols.}

Oxalenphenylhydrazidamidoxim ${ }^{7}$ ),

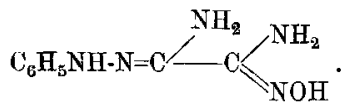

Eine heisse concentrirte alkoholische Lösung von Dicyanphenylhydrazin wird mit ciner möglichst concentrirten wässrigen Lösung von einem Molekül Hydroxylamin (aus dem Chlorhydrat mit Soda bereitet) versetzt. Nach kurzem Erwärmen auf dem Wasserbade beginnt die Abscheidung des in Alkohol schwer löslichen Amidoxims, die nach einigen Minuten beendet ist. Nach dem Abkühlen wird filtrirt und mit Alkohol und Wasser gewaschen. Ausbeute $90-100$ pC. des Ausgangsmaterials. So erhalten, ist das Amidoxim fast rein, zur Analyse wurde es aus Essigäther umkrystallisirt.

$0,2837 \mathrm{~g}$ gaben $0,5142 \mathrm{CO}_{2}$, entsprechend $0,1403 \mathrm{C}$, und $0,1514 \mathrm{H}_{2} \mathrm{O}$, entsprechend $0,0168 \mathrm{H}$.

$0,2175 \mathrm{~g}$ gaben $71,2 \mathrm{ccm}$ Stickgas bei $15,5^{\circ}$ und $713 \mathrm{~mm}$ Druck.

$\begin{array}{ccr} & \text { Berechnct für } & \text { Gefunden } \\ & \mathrm{C}_{8} \mathrm{H}_{11} \mathrm{~N}_{5} \mathrm{O} & \\ \mathrm{C} & 49,74 & 49,46 \\ \mathrm{H} & 5,70 & 5,94 \\ \mathrm{~N} & 36,27 & 36,46\end{array}$

Schöne, stark glänzende Blätter, Schmelzp. $174^{0}$. Sehr schwer löslich in Wasser und Petroläther, etwas melır in Alkohol und Aether, leicht in Essigäther. In ätzenden Alkalien löst sich der Körper als Oxim leicht auf, die Lösung wird indessen durch Oxydation rasch dunkelroth. Ebenso giebt Eisenchlorid in wässriger Lösung eine rothe Färbung.

Acetylderivat. Man löst das Amidoximı in Eisessig und fügt unter Kühlung Essigsäureanhydrid hinzu. Nach Beendigung der Reaction wird vorsichtig mit Wasser und Soda zersetzt,

7) Die Darstellung des Körpers und seines Acetylderivates geschah nach der Vorschrift von Stange. Vergl. Fussnote 3, Seite 129. 
wobei das Acetylderivat krystallinisch ausfällt. Weisse, sehr leicht oxydirbare, daher schwer ganz rein zu erhaltende Blätter aus Alkohol, Schmelzp. $146^{\circ}$. Die Stickstoffbestimmung ergab das Vorliegen eines Monoacetylderivates.

$0,3006 \mathrm{~g}$ gaben $83,1 \mathrm{ccm}$ Stickgas bei $16^{\circ}$ und $716 \mathrm{~mm}$ Druck.

$\begin{array}{ccc} & \text { Berechnet für } & \text { Gefunden } \\ & \mathrm{C}_{8} \mathrm{H}_{10} \mathrm{~N}_{5} \mathrm{O}-\mathrm{COCH}_{9} & \\ \mathrm{~N} & 29,78 & 30,33\end{array}$

Diamidophenylosotriazol, $\mathrm{C}_{6} \mathrm{H}_{\overline{0}}-\mathrm{N}\left\langle\begin{array}{l}\mathrm{N}=\mathrm{C}-\mathrm{NH}_{2} \\ \mathrm{~N}=\mathrm{C}-\mathrm{NH}_{2}\end{array}\right.$.

Die Condensation des Oxalenphenylhydrazidamidoxims zu dem Osotriazol findet am leichtesten durch Erhitzen des Oxims mit der sechsfachen Menge Wasser auf $150^{\circ}$ statt und ist nach etwa zwei Stunden beendigt. Längeres Erhitzen oder höhere Temperaturen beeinflussen die Ausbeute, die zwischen 40 und $50 \mathrm{pC}$. der Theorie schwankt, nicht wesentlich. Der Druck im Autoclaven steigt in Folge von Nebenreactionen, besonders einer bedeutenden Ammoniakabspaltung, auf $20-25 \mathrm{Atm}$. Das Reactionsproduct erstarrt beim Erkalten zu einem Brei von fast ungefärbten, langen Nadeln und einer braunen, leicht schmelzbaren, harzähnlichen Masse. Dasselbe wird nnter Zusatz 5 pC. kohlensaurem Natron zwei Stunden lang mit Dampf destillirt. Dabei gehen Ammoniak, geringe Mengen von Benzol und Phenylhydrazin und mitunter ein stickstofffreier, alkalische Silberlösung nicht reducirender Körper ${ }^{8}$ ) über. Beim Erkalten der noch mit Thierkohle behandelten und vom Harz abfiltrirten Lösung krystallisirt der grösste Theil des Diamidophenylosotriazols in langen, nadelförmigen, fast farblosen Krystallen aus. Der Rest wird der alkalischen Lösung durch Aether entzogen, ein weiterer Antheil ist durch nochmaliges Auskochen des

8) Derselbe scheidet sich in den Vorlagen in gelb gefärbten Kruston ab. Schmelzp. $71^{\circ}$. Seine Zusammensetzung ist ans Materialmangel nicht festgestellt worden. 
Harzes zu gewinnen. Die vereinigten Rohproducte werden in der 20 fachen Menge siedenden Wassers aufgelöst, nochmals mit Thierkohle behandelt und zur Krystallisation gebracht. Die erhaltenen Krystalle sind fast ehemisch rein, der Schmelzpunkt steigt durch wiederholtes Umkrystallisiren aus Wasser nur um wenige Grade und bleibt bei $143^{\circ}$ (uncorr.) constant.

Die alkalische, mit Aether ausgeschüttelte Flüssigkeit enthält im Wesentlichen oxalsaures Natron und Oxyamidophenylosotriazol (siehe unten).

I. $0,1844 \mathrm{~g}$ gaben $0,3705 \mathrm{CO}_{2}$, entsprechend $0,1010 \mathrm{C}$, und 0,0892 $\mathrm{H}_{2} \mathrm{O}$, entsprechend $0,0099 \mathrm{H}$.

$0,1010 \mathrm{~g}$ gaben $36,8 \mathrm{ccm}$ Stickgas bei $13^{\circ}$ und $710 \mathrm{~mm}$ Druek. II. $0,1014 \mathrm{~g} \quad, \quad 0,2047 \mathrm{CO}_{2}$, entsprechend $0,0558 \mathrm{C}$, und 0,0486 $\mathrm{H}_{2} \mathrm{O}$, entsprechend $0,0054 \mathrm{H}$. $0,0922 \mathrm{~g}$ gaben $32,6 \mathrm{cem}$ Stickgas bei $12^{0}$ and $724 \mathrm{~mm}$ Drtick.

\begin{tabular}{ccrc} 
& Berechnet für & $\overbrace{\text { I. }}$ & \multicolumn{1}{c}{ II. } \\
C & $\mathrm{C}_{8} \mathrm{~N}_{\mathbf{5}} \mathrm{H}_{\boldsymbol{8}}$ & 55,01 & 55,04 \\
$\mathrm{H}$ & 54,85 & 5,31 & $\mathbf{5 , 3 2}$ \\
$\mathrm{N}$ & $5, \mathbf{1 4}$ & 40,18 & 39,96
\end{tabular}

Das Diamidophenylosotriazol ist schwer löslich in Wasser oder Ligroïn, leicht löslich in Alkohol, Aether, Chloroform, Benzol. Die wässrige Lösung reagirt neutral und wirkt stark reducirend. Eisenchlorid oder Bromdampf färben selbst sehr verdünnte Jüsungen tief indigblau; ein in eine erwärmte salzsaure Lösung eingetauchter Fichtenspahn wird intensiv roth. Diese Reactionen sind äusserst empfindlich und zum Nachweise geringer Mengen des Diamins geeignet. Das Verhalten gegen Holzfaser ist nur dem Diamidophenylosotriazol und seinen meisten Derivaten eigenthümlich. Diese Reaction, die an die des Pyrrols erinnert, kommt nicht der ganzen Gruppe der Osotriazole zu, wie an anderen von Herrn v. Pechmann freundlichst zur Verfügung gestellten Osotriazolabkömmlingen nachgewiesen wurde.

Alkalische Lösungen des Diamins färben sich an der Luft langsam rothbraun. Beim Erhitzen alkalischer Lösungen auf 
$150-200^{\circ}$ entsteht ein rothbraunes, unschmelzbares, in fast allen gebräuchlichen Lösungsmitteln unlösliches Condensationsproduct. Dasselbe färbt concentrirte Schwefelsäure dunkelblau. Behandelt man das Diamidophenylosotriazol mit schmelzendem Kali, so findet nur eine sehr langsame und theilweise Zersetzung unter Ammoniakabspaltung statt. Der grösste Theil des Diamins kann unverändert der Schmelze wieder durch Aether entzogen werden. Ebenso sind nach längerem Erhitzen der Base mit rauchender Salzsäure auf $150^{\circ}$ im Reactionsproducte nur geringe Mengen von Chlorammonium nachweisbar. Erst bei Temperaturen über $200^{\circ}$ findet eine totale Zersetzung statt. Phenylprozan,

$$
\mathrm{C}_{6} \mathrm{H}_{5}-\mathrm{N}<\frac{\mathrm{NH}_{2}}{\mathrm{NH}_{2}{ }^{\prime}}
$$

konnte in keinem Falle isolirt werden.

Chlorhydrat des Diamidophenylosotriazols, $\quad \mathrm{C}_{8} \mathrm{~N}_{5} \mathrm{H}_{9} \cdot \mathrm{HCl}$. Dasselbe krystallisirt aus Wasser oder verdünntem Alkohol in farblosen Blättchen, deren wässrige Lösung leicht dissociirt. Durch häufiges Umkrystallisiren aus Wasser wird zuletzt die reine Base erhalten. Versuche, ein Salz derselben mit zwei Molekülen $\mathrm{HCl}$ darzustellen, gelangen nicht. Beim Einleiten von trocknem Chlorwasserstoff in eine ätherische Lösung des Diamins wurde ein Chlorhydrat erhalten, das, mit Aether gewaschen und über Natronkalk getrocknet, annähernd die dem cinfachen Chlorhydrat entsprechende Menge Chlor enthielt. Schmelzp. $210^{\circ}$ (unter Zersetzung).

$0,3343 \mathrm{~g}$ gaben $0,2333 \mathrm{AgCl}$, entsprechend 0,0589 $\mathrm{HCl}$.

Berechnet für $\mathrm{C}_{8} \mathrm{H}_{9} \mathrm{~N}_{5} \cdot \mathrm{HCl}$

HCl 17,21
Gefunden

17,62

Pikrat, $\mathrm{C}_{8} \mathrm{~N}_{5} \mathrm{H}_{9} \cdot \mathrm{C}_{6} \mathrm{H}_{2}\left(\mathrm{NO}_{2}\right)_{3} \mathrm{OH}$. Dasselbe fällt in gelben, büschelförmigen, in heissem Wasser löslichen Nadeln aus, wenn man eine Lösung des salzsauren Diamins mit einer gesättigten, wässrigen Pikrinsäurelösung versetzt. Schmelzp. $153^{\circ}$.

$0,1197 \mathrm{~g}$ gaben $29 \mathrm{ccm}$ Stickgas bei $8^{0}$ und $729 \mathrm{~mm}$ Druck. 


$\begin{array}{ccc} & \text { Berechnet für } & \text { Gefunden } \\ & \mathrm{C}_{\mathbf{1 4}} \mathrm{H}_{\mathbf{1 0}} \mathrm{N}_{8} \mathrm{O}_{7} & \\ \mathrm{~N} & 27,72 & 28,05\end{array}$

Sulfat, $\left(\mathrm{C}_{8} \mathrm{~N}_{5} \mathrm{H}_{9}\right)_{2} \cdot \mathrm{H}_{2} \mathrm{SO}_{4}$. Fügt man zu einer alkoholischen oder wässrigen Lösung des Diamidophenylosotriazols verdünnte Schwefelsäure, so wird das Sulfat in farblosen, blättrig verzweigten, tafelförmigen Krystallen ausgescbieden. Dieselben sind sehr schwer löslich in kaltem, etwas leichter löslich in heissem Wasser. Sie färben sich an der Luft oder beim Umkrystallisiren schwach gelb und zersetzen sich beim Erhitzen, ohne zu schmelzen. Aus der Analyse ergiebt sich die Zusammensetzung $\left(\mathrm{C}_{8} \mathrm{~N}_{6} \mathrm{H}_{9}\right)_{2} \mathrm{H}_{2} \mathrm{SO}_{4}$.

$0,1616 \mathrm{~g}$ gaben $0,2523 \mathrm{CO}_{2}$, entsprechend $0,0688 \mathrm{C}$, und $0,0682 \mathrm{H}_{2} \mathrm{O}$, entsprechend $0,0076 \mathrm{H}$.

$0,1100 \mathrm{~g}$ gaben $30,6 \mathrm{~cm}$ Stickgas bei $13^{9}$ und $727 \mathrm{~mm}$ Druck.

$0,5654 \mathrm{~g} \quad, \quad 0,2982 \mathrm{BaSO}_{4}$, entsprechend $0,0409 \mathrm{~s}$.

Berechnet für Gefunden $\left(\mathrm{C}_{8} \mathrm{~N}_{5} \mathrm{H}_{9}\right)_{2} \mathrm{SO}_{4} \mathrm{H}_{2}$

$\begin{array}{lrr}\mathrm{C} & 42,85 & 42,57 \\ \mathrm{H} & 4,46 & 4,68 \\ \mathrm{~N} & 31,25 & 31,42 \\ \mathrm{~S} & 7,14 & 7,24\end{array}$

Diamidophenylosotriazol-Silbernitrat, $\mathrm{C}_{8} \mathrm{~N}_{5} \mathrm{H}_{9} \cdot \mathrm{AgNO}_{3}$. Bei der Einwirkung von Silbernitrat auf das Diamidophenylosotriazol kann das erste leicht zersetzliche Einwirkungsproduct, das auf ein Molekül Diamin ein Molekül Silbernitrat enthält, isolirt werden, wenn man eine mit Salpetersäure schwach angesäuerte Lösung mit einer concentrirten Silbernitratlösung versetzt, den Niederschlag rasch absaugt, mit Alkohol, dann mit Aether wäscht und trocknet. Die Verbindung krystallisirt in farblosen Blättchen, die unter Schwarzfärbung und totaler Zersetzung bei $150^{\circ}$ schmelzen. Sie oxydirt sich in trocknem Zustande nur langsam, ist kaum löslich in Wasser, löslich in verdünnten Säuren und Essigsäure. Die Analyse ergab die von der obigen Formel verlangten Werthe. 


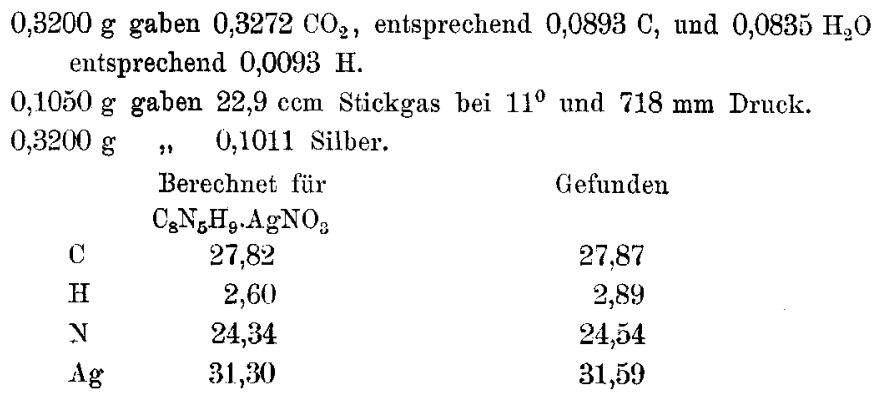

Saure Lösungen der Silberverbindung färben sich nach kurzer Zeit blau. Das entstebende Oxydationsproduct dürfte mit dem aus dem Diamin und Eisenchlorid erhaltenen Azin identisch sein.

Diamidophenylosotriazolsulfosäure, $\mathrm{SO}_{3} \mathrm{H}-\mathrm{C}_{6} \mathrm{H}_{4} \mathrm{~N} \prod_{\mathrm{N}=\mathrm{CNH}_{2}}^{\mathrm{N}=\mathrm{CNH}_{2}}$

Die Sulfurirung des Diamins geschieht bei $200-220^{\circ}$ oder einfacher durch Anflösen der Base in acht- bis zehnprocentigcr rauchender Schwefelsäure. Die sich stark erwärmende Lösung wird nach einigen Minuten, wenn eine Probe die Eisenchloridreaction des Diamins nicht mehr zeigt, in kaltes Wasser eingegossen. Dabei scheidet sich die Sulfosäure nahezu quantitativ aus. Der Niederschlag wird abgesaugt, mit heissem Wasser gewaschen, in Ammoniak gelöst und nochmals mit Schwefelsäure gefällt. Mit Alkohol, dann mit Aether gewaschen und im Toluolbade bis z.ur Gewichtsconstanz getrocknet, ist das Präparat analysenrein.

$0,2113 \mathrm{~g}$ gaben $0,2921 \mathrm{CO}_{2}$, entsprechend $0,07966 \mathrm{C}$, und $0,0688 \mathrm{H}_{2} \mathrm{O}$, entsprechend $0,00764 \mathrm{H}$.

$0,2182 \mathrm{~g}$ gaben $53 \mathrm{ccm}$ Stickgas bei $13^{\circ}$ und $726 \mathrm{~mm}$ Druck.

$0,4680 \mathrm{~g} \quad, \quad 0,4200 \mathrm{BaSO}_{4}$, entsprechend $0,0576 \mathrm{~s}$.

$\begin{array}{ccr} & \text { Berechnet für } & \text { Gefunden } \\ & \mathrm{C}_{\mathrm{S}} \mathrm{H}_{3} \mathrm{~N}_{5} \mathrm{SO}_{3} & \\ \mathrm{C} & 37,63 & 37,70 \\ \mathrm{H} & 3,52 & 3,61 \\ \mathrm{~N} & 27,44 & 27,40 \\ \mathrm{~S} & 12,54 & 12,30\end{array}$


Farblose, nadelförmige Krystalle, welche in Wasser äusserst schwer löslich sind und beim Erhitzen auf höhere Temperaturen, ohne zu schmelzen, verkohlen. Wässrige Lösungen, die Spuren der Sulfosäure enthalten, werden durch sehr verdünnte Bromlösungen intensiv braun gefärbt. Die Farbe verschwindet auf Zusatz von Mineralsäuren nicht, wird dagegen durch Aetzalkalien zerstört. Mit Eisenchlorid reagirt die Sulfosäure nicht. Wit Alkalien entstehen schwer lösliche Salze. Das Natriumsalz krystallisirt in farblosen Tafeln. Das Ammoniaksalz ist leicht löslich und kann aus beissem Wasser in schönen, farblosen Nadeln erhalten werden. Es schmilzt nicht und ist schwer verbrennbar.

$0,1392 \mathrm{~g}$ gaben $37,6 \mathrm{~cm}$ Stickgas bei $10,5^{0}$ und $717 \mathrm{~mm}$ Druck.

$\begin{array}{ccc} & \text { Berechnet für } & \text { Gefunden } \\ & \mathrm{C}_{8} \mathrm{H}_{3} \mathrm{~N}_{5} \mathrm{SO}_{3} \cdot \mathrm{NH}_{3} & \\ \mathrm{~N} & 30,88 & 30,35\end{array}$

Das Silbersalz ist ein in Ammoniak leicht löslicher, weisser Niederschlag, dessen ammoniakalische Lösung bei gelindem Erwärmen reducirt wird.

\section{Azin aus Diamidophenylosotriazol,}

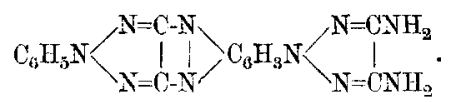

$5 \mathrm{~g}$ Diamidophenylosotriazol werden in $400 \mathrm{~g}$ einprocentiger Salzsäure gelöst und mit einer Lösung von $10 \mathrm{~g}$ wasserhaltigem Eisenchlorid in $100 \mathrm{~g}$ Wasser versetzt. Der blaue, amorphe, sehr fein vertheilte Niederschlag wird abfiltrirt, mit viel Wasser gewaschen, abgesaugt und auf Thontellern getrocknet. Wegen seiner Unlöslichkeit in fast allen Lösungsmitteln konnte er nicht in krystallisirtem Zustande erhalten werden. Alkohol Iöst zwar geringe Mengen mit grüner Farbe auf, indessen zersetzen sich diese Lösungen sehr bald unter Bräunung und Entwickelung von Blausäure. Die Lösung des Azins in concentrirter Schwefelsäure ist tiefblau.

Fügt man zu dem frisch gefällten, noch feuchten Farbstoff Zinnchlorür und Salzsäure, so verschwindet die blaue Farbe 


\section{Thiele u. Schleussner, Diamidophenylosotriazol.}

beim Erwärmen auf dem Wasserbade vollständig. Die Flüssigkeit wird röthlichgelb und enthält die Leukoverbindung in äusserst feiner Vertheilung suspendirt. Bei Zusatz von sehr viel siedendem Wasser geht der grösste Theil des Niederschlags in Lösung und scheidet sich beim Erkalten in sehr feinen, büschelförmig geordneten, farblosen Nädelchen aus, die sich an der Luft leicht oxydiren. Man extrahirt daher zweckmässiger mit Aether und krystallisirt den schwach gelbbraun gefärbten Aetherrückstand wiederholt aus verdünntem Alkohol um. Die Analyse der auf diese Weise erhaltenen, nicht ganz farblosen Leukoverbindung führte zu der Formel:

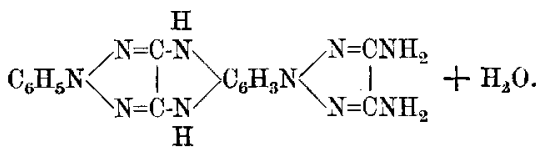

$0,2168 \mathrm{~g}$, im Toluolbade getrocknet, gaben $0,4396 \mathrm{CO}_{2}$, entsprechend

$0,11989 \mathrm{C}$, and $0,0901 \mathrm{H}_{2} \mathrm{O}$, entsprechend $0,01001 \mathrm{H}$.

$0,1271 \mathrm{~g}$ gaben $45,4 \mathrm{ccm}$ Stickgas bei $14^{\circ}$ und $726 \mathrm{~mm}$ Druck.

$\begin{array}{ccr} & \text { Berechnet für } & \text { Gefunden } \\ & \mathrm{C}_{16} \mathrm{~N}_{10} \mathrm{H}_{14} & \\ \mathrm{C} & 55,46 & 55,29 \\ \mathrm{H} & 4,05 & 4,61 \\ \mathrm{~N} & 40,46 & 40,12\end{array}$

$0,4375 \mathrm{~g}$, wasserhaltio, verloren im Toluolbade $0,0225 \mathrm{~g}$.

$$
\begin{array}{ccc} 
& \begin{array}{c}
\text { Berechnet für } \\
\mathrm{C}_{10} \mathrm{~N}_{10} \mathrm{H}_{14}+\mathrm{H}_{2} \mathrm{O}
\end{array} & \text { Gefunden } \\
\mathrm{H}_{3} \mathrm{O} & 4,94 & 5,14
\end{array}
$$

Die Leukoverbindung des Azins ist in Alkohol, Aether, Chloroform oder heissem Benzol leicht löslich, schwer löslich in kaltem, leichter in kochendem Wasser. Die wasserfreie Verbindung schmilzt bei $175^{\circ}$. Wird die wasserhaltige Substanz rasch über $120^{\circ}$ erhitzt, so findet man den Schmelzpunkt bei $128^{\circ}$; bei weiterem Erhitzen wird das Präparat wieder fest, um zwischen $180-185^{\circ}$ unscharf zu schmelzen. Durch Oxydationsmittel wird der blaue Farbstoff regenerirt. 
Chinoxalin aus Diamidophenylosotriazol und Benzil, $\mathrm{C}_{6} \mathrm{H}_{5} \mathrm{~N} \prod_{\mathrm{N}=\mathrm{C}-\mathrm{N}=\mathrm{C}-\mathrm{C}_{6} \mathrm{H}_{5}}^{\mathrm{N}=\mathrm{C}-\mathrm{N}=\mathrm{C}-\mathrm{C}_{6} \mathrm{H}_{5}}$.

Dasselbe entsteht durch Erhitzen molekularer Mengen der Componenten in Eisessiglösung. Aus Eisessig krystallisirt es in ziegelrothen, derben Nadeln vom Schmelzp. 217 ${ }^{\circ}$. Es ist unlöslich in Säuren, Alkalien, Ammoniak, kaum löslich in Wasser, leicht löslich in Eisessig, Chloroform, Aether oder Benzol. Mit concentrirter Schwefelsäure geben die geringsten Mengen eine intensiv violette Färbung, die auf Zusatz von wenig Wasser in Roth übergeht und bei weiterer Verdünnung unter Trübung verschwindet.

$0,1210 \mathrm{~g}$ gaben $22 \mathrm{~cm}$ Stickgas bei $14^{0}$ und $715,5 \mathrm{~mm}$ Druek.

$$
\begin{array}{ccc} 
& \text { Berechnet fiir } & \text { Gefunden } \\
& \mathrm{C}_{2: 2} \mathrm{H}_{15,5} \mathrm{~N}_{5} & \\
\mathrm{~N} & 20,05 & 20,11
\end{array}
$$

Chinoxalin aus Diamidophenylosotriazol und Phenanthrenchinon,

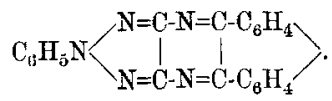

Kocht man Diamidophenylosotriazol mit Phenanthrenchinon und Eisessig, so scheidet sich das Chinoxaliu schon in der Siedehitze in bräunlichgelben Nadeln aus. Dieselben schmelzen bei $289^{\circ}$, sind unlöslich in Wasser, Säuren, Alkalien, sehr sehwer löslich in Alkohol, Eisessig oder Benzol. Sie lösen sich leichter in heissem Anilin oder Toluol und werden aus diesen Lösungen auf Zusatz von Aether oder Ligroin wieder ausgeschieden. Zum Umkrystallisiren eignet sich am besten siedendes Chloroform.

$0,1278 \mathrm{~g}$ gaben $23,4 \mathrm{ccm}$ Stickgas bei $15^{\circ}$ und $721 \mathrm{~mm}$ Druck.

$$
\begin{array}{ccc} 
& \text { Berechnet für } & \text { Gefunden } \\
& \mathrm{C}_{22} \mathrm{H}_{13} \mathrm{~N}_{5} & \\
\mathrm{~N} & 20,17 & 20,32
\end{array}
$$

Das Chinoxalin färbt concentrirte Schwefelsäure tief indigoblau. 
Thiele u. Schleussner, Diamidophenylosotriazol.

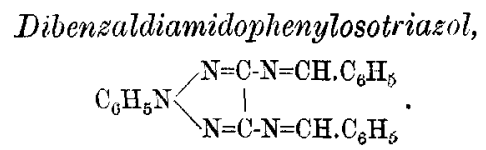

Eine Lösung von $2 \mathrm{~g}$ Diamidophenylosotriazol in $5 \mathrm{ccm}$ Eisessig wird mit $2^{1 / y}$ g Benzaldehyd versetzt und zum Sieden erhitzt. Beim Erkalten erstarrt das Reactionsproduct zu einem Brei von gelben, prismatischen Krystallen. Dieselben werden abgesaugt, mit wenig Alkohol gewaschen und aus Eisessig oder Toluol umkrystallisirt. Schmelzp. $162^{\circ}$.

$0,1908 \mathrm{~g}$ gaben $0,5248 \mathrm{CO}_{2}$, entsprechend $0,1.4313 \mathrm{C}$ und $0,0880 \mathrm{H}_{2} \mathrm{O}$, entsprechend $0,00977 \mathrm{H}$.

$0,1810 \mathrm{~g}$ gaben $32,6 \mathrm{ccm}$ Stickgas bei $12^{\circ}$ and $721 \mathrm{~mm}$ Druck.

$\begin{array}{ccr} & \text { Berechnet für } & \text { Qefunden } \\ & \mathrm{C}_{\mathbf{2} 2} \mathrm{H}_{\mathbf{1 9}} \mathrm{N}_{\overline{\mathbf{a}}} & \\ \mathrm{C} & \mathbf{7 5 , 2 1} & 75,01 \\ \mathrm{H} & 4,84 & \check{0}, 13 \\ \mathrm{~N} & 19,94 & 20,26\end{array}$

Die Verbindung ist unlöslich in Alkalien, kohlensaurem Alkali, Ammoniak, Essigsäure und verdünnten Mineralsäuren. In Alkohol oder Aether ist sie schwer löslich, leichter in Benzol oder Essigäther, sehr leicht in Chloroform oder siedendem Toluol. Säuren spalten schon in der Kälte, leicht beim Erwärmen Benzaldehyd ab. Das hierbei regenerirte Diamin wurde - nach Entfernung des Benzaldehyds - durch Ausäthern der alkalisch gemachten Lösung isolirt und durch seine Reactionen identificirt.

Es ist nicht gelungen, das Dibenzaldiamidophenylosotriazol in die isomere Anhydrobase

umzulagern.

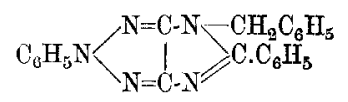

Dioxybenzaldiamidophenylosotriazol,

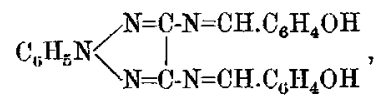

wird in analoger Weise wie das Dibenzaldiamidophenylosotriazol aus Diamidophenylosotriazol und Salicylaldehyd dargestellt. Es 
krystallisirt in gelben Nadeln, ist alkalilöslich und schmilzt bei $210^{\circ}$.

$0,1169 \mathrm{~g}$ gaben $19 \mathrm{~cm}$ Stickgas bei $12^{\prime \prime}$ und $718 \mathrm{~mm}$ Druck.

$\begin{array}{ccc} & \text { Berechnet für } & \text { Ciefunden } \\ & \mathrm{C}_{22} \mathrm{~N}_{5} \mathrm{H}_{17} \mathrm{O}_{2} & \\ \text { N } & 18,27 & 18,19\end{array}$

Monoacetyldiamidophenylosotriazol, $\mathrm{C}_{6} \mathrm{H}_{5} \mathrm{~N} \underset{\mathrm{N}=\mathrm{CNH}_{2}}{\mathrm{~N}=\mathrm{CNH} . \mathrm{CO} \cdot \mathrm{CH}_{3}}$

Wenn man Diamidophenylosotriazol mit Eisessig am Rückflusskühler kocht, bis eine Probe verdünnte Eisenchloridlösung gar nicht oder nur schwach blau fürbt, so erhält man im Wesentlichen Monoacetyldiamidophenylosotriazol. Demselben sind je nach der Dauer des Erhitzens wechselnde Mengen von Diacetyldiamidophenylosotriazol beigemischt. Die Trennung kann durch häufiges Umkrystallisiren aus Wasser, in dem die Diacetylverbindung schwerer löslich ist, bewirkt werden. Farblose Nadeln vom Schmelzp. $186^{\circ}$.

Einfacher und zweckmässiger ist die Darstellung des Monoacetyldiamidophenylosotriazols durch Zusammenschmelzen molekularer Mengen von Diacetyldiamidophenylosotriazol und Diamidophenylosotriazol und drei- bis vierstündiges Erhitzen im Oelbade auf $200-210^{\circ}$. Die erkaltete Schmelze wird in Alkohol gelöst, die alkoholische Lösung mit Thierkohle entfärbt und nach dem Verdunsten des grössten Theils des Alkohols in kaltes Wasser gegossen. Dabei scheidet sich die Monoacetylverbindung fast rein aus. Die Krystalle werden abgesaugt und aus verdünntem Alkohol umkrystallisirt. Das so erhaltene Monoacetylderivat ist zwar meistens nicht ganz farblos, im Gegensatze zu dem durch directes Acetyliren der Base erhaltenen, doch ist es zur weiteren Verarbeitung genügend rein.

I. $0,2754 \mathrm{~g}$ gaben $0,5596 \mathrm{CO}_{2}$, entsprechend $0,1526 \mathrm{c}$, und $0,1396 \mathrm{H}_{2} \mathrm{O}$, cutsprechend $0,01518 \mathrm{H}$.

$0,1204 \mathrm{~g}$ gaben $34,6 \mathrm{ccm}$ Stickgas bei $14^{\circ}$ und $722 \mathrm{~mm}$ Druck. 
II. $0,1738 \mathrm{~g}$ gaben $0,3501 \mathrm{CO}_{2}$, entsprechend $0,09548 \mathrm{C}$, und 0,0891 $\mathrm{I}_{2} \mathrm{O}$, entsprechend $0,0091 \mathrm{H}$.

$0,1485 \mathrm{~g}$ gaben $43 \mathrm{ccm}$ Stickgas bei $14^{0}$ und $726 \mathrm{~mm}$ Druck.

\begin{tabular}{|c|c|c|}
\hline \multirow{2}{*}{$\begin{array}{c}\text { Berechnet fïr } \\
\mathrm{C}_{10} \mathrm{H}_{11} \mathrm{~N}_{5} \mathrm{O}\end{array}$} & \multicolumn{2}{|c|}{ Gefunden } \\
\hline & I. & II. \\
\hline 55,29 & 55,41 & 54,93 \\
\hline 5,06 & 5,51 & 5,23 \\
\hline 32,25 & 32,09 & 32,52 \\
\hline
\end{tabular}

Das Monoacetyldiamidophenylosotriazol ist ziemlich leicht löslich in Alkalien, unlöslich in verdünnten Säuren oder Ammoniak, durch Kochen mit Säuren oder Alkalien wird es gespalten. Aus der Lösung in verdünnter Natronlauge wird es durch Einleiten von Kohlensäure wieder ausgefällt. Unlöslich in Petroläther, schwer in Aether und Benzol, leicht in Essigäther.

Mit Eisenchlorid reagirt es nicht; Blaufärbung tritt erst durch Erhitzen mit Eisenchlorid und verdünnten Mineralsäuren ein, das heisst, nach Abspaltung des Acetylrestes und Regenerirung des Diamins.

Die Abspaltung von Wasser zwischen der Amidogruppe und dem Acetylrest unter Bildung eines Imidazols,

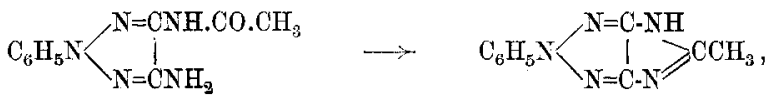

konnte weder durch längeres Erhitzen der Monoacetylverbindung für sich, noch durch Schmelzen mit wasserentziehenden Mitteln bewirkt werden. Bei Anwendung von Chorzink entsteht eine tief violette, in Wasser schwer, in Alkohol leicht lösliche Schmelze.

Salpetrige Säure fübrt die Acetylverbindung leicht in eine Diazoverbindung über, welche mit R-Salz in Sodalösung intensiv blauroth kuppelt. (Vergl. unten.)

Diacetyldiamidophenylosotriazol,<smiles></smiles>

Die Acetylirung der beiden Amidogruppen des Diamidophenylosotriazols verläuft glatt und quantitativ, wenn man das 
Diamin mit einem kleinen Ueberschusse von Essigsäureanhydrid zum Sieden erhitzt. Nach wenigen Minuten scheiden sich kurze, farblose, prismatische, in Alkobol leicht, in kaltem Wasser sehr schwer lösliche Krystalle aus. Schmelzp. 2060. Das Diacetyldiamidophenylosotriazol färbt Eisenchloridlösung auch beim Erwärmen nicht blau. Es ist unlöslich in verdünnten Säuren und Ammoniak, löslich in verdünnter Natronlauge. Durch Kohlensäure wird es ebenso wie das Monoacetylderivat wieder ausgefällt.

$0,1819 \mathrm{~g}$ gaben $0,3722 \mathrm{CO}_{2}$, entsprechend $0,1015 \mathrm{C}$, und $0,0794 \mathrm{H}_{2} \mathrm{O}$, entsprechend $0,00882 \mathrm{H}$.

$0,1385 \mathrm{~g}$ gaben $32,8 \mathrm{~cm}$ Stickgas bei $10^{\circ}$ und $724 \mathrm{~mm}$ Druck.

$\begin{array}{ccr} & \text { Berechnet für } & \text { Gefunden } \\ & \mathrm{C}_{12} \mathrm{H}_{19} \mathrm{~N}_{5} \mathrm{O}_{2} & \\ \mathrm{C} & \tilde{5} 5,59 & 55,79 \\ \text { II } & 5,01 & 4,84 \\ \mathrm{~N} & 27,02 & 27,00\end{array}$

In ein Imidazol liess sich das Diacetylderivat ebensowenig umwandeln, wie das Monoacetylderivat.

\section{Dibenzoyldiamidophenylosotriazol,}

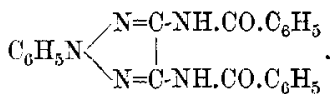

Kocht man ein Molekül Diamidophenylosotriazol (2 g) und zwei Moleküle Benzoylchlorid $(3,5 \mathrm{~cm})$ mit wenig Wasser, so erfolgt sofort Ausscheidung der Dibenzoylverbindung. Dieselbe ist unlöslich in Wasser, schwer löslich in heissem Alkohol, Eisessig oder Chloroform, unlöslich in Petroläther. Kalte verdünnte Natronlauge löst schwer, Mineralsäuren lösen das Dibenzoyldiamidophenylosotriazol nicht. Die tafelförmigen, sehr kleinen Krystalle schmelzen bei $242^{\circ}$.

$0,1884 \mathrm{~g}$ gaben $0,4744 \mathrm{CO}_{2}$, cntsprechend $0,12938 \mathrm{C}$, und $0,0780 \mathrm{H}_{2} \mathrm{O}$, entsprechend $0,00866 \mathrm{HI}$.

$0,1996 \mathrm{~g}$ gaben $33 \mathrm{ccm}$ Stickgas bei $14^{\circ}$ und $722 \mathrm{~mm}$ Druck.

Berechnet für $\mathrm{C}_{22} \mathrm{~N}_{5} \mathrm{H}_{17} \mathrm{O}_{2}$

$\mathrm{C}$

$\mathrm{H}$

$\mathrm{N}$
68,93

4,43

18,27
Gefunden

68,67

$18, \% 5$ 
Durch Erhitzen mit concentrirter Salzsäure ${ }^{8}$ ) auf $170^{\circ}$ konnte das entsprechende Imidazol nicht erhalten werden. Die Dibenzoylverbindung ging in das Chlorhydrat des Diamins über.

$$
\begin{aligned}
& \text { Amidodiazophenylosotriazol, } \\
& \mathrm{C}_{6} \mathrm{H}_{5} \mathrm{~N} \backslash \begin{array}{c}
\mathrm{N}=\mathrm{C}-\mathrm{N}_{2} \mathrm{OH} \\
1 \\
\mathrm{~N}=\mathrm{C}-\mathrm{NH}_{2}
\end{array} .
\end{aligned}
$$

Die Diazotirung des Diamidophenylosotriazols gelingt glatt bei Einhaltung der folgenden Versuchsbedingungen:

5 g Diamidophenylosotriazol werden unter Zusatz von $5 \mathrm{ccm}$ concentrirter Salzsäure in $200 \mathrm{ccm}$ Wasser gelöst und dic Lösung unter gutem Umrühren (mit Hülfe eines Witt'scheı Rührers) in eine stark abgekühlte Lösung von $4 \mathrm{~g}$ Natriumnitrit in $300 \mathrm{ccm}$ Wasser und $20 \mathrm{ccm}$ concentrirter Salzsäure eingetropft. Wenn das Amin vollständig eingetragen ist, wird die Diazolösung sofort von einem in geringer Menge gebildeten Niederschlage abfiltrirt. Sie ist dunkelgelb gefärbt und wird nach einiger Zeit trübe. Fin Ueberschuss von salpetriger Säure wirkt nicht schädlich.

Lässt man umgekehrt die Nitritlösung in eine saure Lösung der Base einfliessen, so entsteht in nicht unbeträchtlicher Menge ein amorpher, grünlichgelber, unlöslicher Körper, muthmasslich ein Oxydationsproduct.

Das Amidodiazophenylosotriazol besitzt im Gegensatze zu den meisten Diazoverbindungen eine ziemlich grosse Beständigkeit. Lösungen in verdünnten Mineralsäuren können kurze Zeit gekocht oder ein bis zwei Stunden auf dem Wasserbade erwärmt werden, ohne die Fähigkeit, mit Aminen und Phenolen zu kuppeln, zu verlieren. Es theilt diese Eigenschaft mit einigen Diazokörpern, welche stark negative Radicale enthalten (z. B. Diazotribrombenzol ${ }^{9}$ )). Bemerkenswerth ist die Leichtigkeit, mit der es sich in die Isodiazoform umlagert, ein Ver-

8) Brückner, diese Annalen $\mathbf{2 0 5}, 117$.

9) Silberstein, Journ. f. pract. Chem. 27, 98. 
halten, das von Thiele und Ingle auch bei dem Diazotetrazol beobachtet wurde ${ }^{10}$ ).

\section{Amidodiazophenylosotriazolpikrat,}

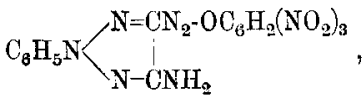

fällt in Form von gelben, in heissem Wasser leicht löslichen Nadeln aus, wenn man die Diazolösung mit einer gesättigten, wässrigen Pikrinsäurelösung versetzt. Dieselben schmelzen bei $121^{\circ}$ unter Zersetzung und sind weder durch Schlag noch durch Stoss zur Explosion zu bringen. Bei raschem Erhitzen erfolgt schwaches Verpuffen.

$0,2017 \mathrm{~g}$ gaben $0,2996 \mathrm{CO}_{2}$, entsprechend $0,08173 \mathrm{C}$, und $0,0454 \mathrm{H}_{2} \mathrm{O}$, entsprechend $0,00504 \mathrm{H}$.

$0,1220 \mathrm{~g}$ gaben $33,2 \mathrm{~cm}$ Stickgas bei $16^{\circ}$ und $717 \mathrm{~mm}$ Druck.

Berechnet für Gefunden

$\begin{array}{lcr} & \mathrm{C}_{\mathbf{1 4}} \mathrm{N}_{\mathbf{9}} \mathrm{H}_{9} \mathrm{O}_{7} & \\ \mathrm{C} & 40,48 & 40,51 \\ \mathrm{H} & 2,16 & 2,49 \\ \mathrm{~N} & 30,36 & 29,90\end{array}$

Zum weiteren Nachweise, dass bei der Einwirkung überschüssiger salpetriger Säure nur eine Amidogruppe des Diamins diazotirt wird, wurde das Diazoamidophenylosotriazol mit Dimethylanilin gekuppelt. Dabei entsteht:

Dimethylanilinazoamidophenylosotriazol, $\mathrm{C}_{6} \mathrm{H}_{5} \mathrm{~N}\left\langle\begin{array}{l}\mathrm{N}=\mathrm{C}-\mathrm{N}=\mathrm{N}-\mathrm{C}_{6} \mathrm{H}_{4} \mathrm{~N}\left(\mathrm{CH}_{3}\right)_{2} \\ \substack{\mathrm{N}=\mathrm{CNH} \\ 2}\end{array}\right.$.

Die Kuppelung geschieht durch Eingiessen der mit Natriumacetat versetzten Diazolösung in die mit Wasser verdünnte Lösung des Dimethylanilins in Eisessig. Dabei scheidet sich der Azofarbstoff quantitativ als zinnoberrother, flockiger Niederschlag aus. Er wird auf einer Filterplatte gesammelt, mit Wasser gut ausgewaschen und aus heissem Alkohol umkrystalli-

10) Thiele und Ingle, diese Annalen 287, 244, Anm. 
sirt. Rothe, in kaltem Alkohol sehr schwer lösliche Nadeln vom Schmelzp. $243^{\circ}$ (Zersetzung).

$0,220 亏 \mathrm{~g}$ gaben $0,5043 \mathrm{CO}_{2}$, entsprechend $0,13754 \mathrm{C}$, und $0,1152 \mathrm{H}_{2} \mathrm{O}$, entsprechend $0,01280 \mathrm{H}$.

$0,1219 \mathrm{~g}$ gaben $34,4 \mathrm{ccm}$ Stickgas bei $10^{\circ}$ und $725 \mathrm{~mm}$ Druck.

$\begin{array}{ccr} & \text { Berechnet fuir } & \text { Gefunden } \\ & \mathrm{C}_{10} \mathrm{H}_{1 \mathbf{2}} \mathrm{N}_{7} & \\ \mathrm{C} & 62,54 & 62,37 \\ \mathrm{H} & 5,53 & \tilde{\mathbf{5}}, 80 \\ \mathrm{~N} & 31,92 & 32,21\end{array}$

Der Farbstoff färbt Wolle orangegelb. Mit concentrirter Schwefelsäure giebt er eine intensiv cochenillerothe Lösung, die sich auch beim Erwärmen nicht verändert.

\section{Phenylosotriazolazimid,}

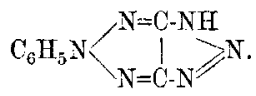

Versetzt man die salzsaure Lösung des Amidodiazophenylosotriazols mit essigsaurem Natron und erwärmt auf dem Wasserbade, so verschwindet die Reaction auf Diazoverbindung. Die Flüssigkeit trübt sich stark und scheidet nach Verlauf einiger Stunden das Azimid als voluminösen, gelb gefärbten Niederschlag ab. Dasselbe wird bcim Schüteln mit Aether von diesem fast vollständig aufgenommen und bleibt beim Verdunsten des Aethers als gelb bis braun gefürbte Masse zurück.

Das Präparat wird reiner und in besserer Ausbeute erhalten, wenn sich die Anhydridbildung nur langsam vollzieht. Man lässt die salzsaure Diazolösung bei gewöhnlicher Temperatur unter häufigem Umschütteln so lange stchen, bis cine Probe mit R-Salz nicht mehr kuppelt. Die Umsetzung ist gewöhnlich in sechs bis acht Tagen vollendet. Die gelblichen, mit kaltem Wasser gewaschenen Krystalle werden in verdünnter Sodalösung aufgelöst. Auf Zusatz von verdünnter Salzsäure fällt das Phenylosotriazolazimid fast roin aus.

Am einfachsten und glattesten ist die Darstellung aus 
dem weiter unten zu beschreibenden Phenylosotriazolacetylazimid durch Abspaltung des Acetylrestes.

Das Acetylazimid wird mit Natronlauge bis zur Lösung erwärmt, die klare, schwach braun gefärbte Flüssigkeit mit Wasser verdünnt und mit Thierkohle entfärbt. Der beim Ansäuern mit Salzsäure sich ausscheidende Niederschlag wird abgesaugt, mit Wasser gewaschen und aus viel heissem Wasser umkrystallisirt.

I. $0,1942 \mathrm{~g}$ gaben $0,3678 \quad \mathrm{CO}_{2}$, entsprechend $0,10031 \mathrm{c}$, und $0,0600 \mathrm{H}_{2} \mathrm{O}$, entsprechend $0,0067 \mathrm{H}$.

$0,1044 \mathrm{~g}$ gaben $42,2 \mathrm{ccm}$ Stickgas bei $12^{\circ}$ und $720 \mathrm{~mm}$ Druck. II. $0,0786 \mathrm{~g} \quad, \quad 31,8 \mathrm{~cm}$ Stickgas, $11^{\circ}$, $717 \mathrm{~mm}$ Druek.

\begin{tabular}{ccrc} 
& Berechnet fïr & \multicolumn{2}{c}{ Gefunden } \\
\cline { 3 - 4 } & $\mathrm{C}_{5} \mathrm{H}_{6} \mathrm{~N}_{6}$ & I. & II. \\
$\mathrm{C}$ & 51,61 & 51,64 & - \\
$\mathrm{H}$ & 3,22 & 3,43 & -- \\
$\mathrm{N}$ & 45,16 & 45,42 & 45,47
\end{tabular}

Das Phenylosotriazolazimid löst sich sehr leicht in Alkohol, Aether, Chloroform oder heissem Benzol. In kaltem Wasser ist es schwer, in heissem Wasser leichter löslich, in Ligrö̈n unlöslich. Aus Wasser krystallisirt es in farblosen Nadeln, die sich an der Luft allmählich schwach gelb färben. Es bräunt sich beim Erhitzen auf $130-140^{\circ}$ und verpufft unter Schmelzen zu einer schwarzen Masse bei $147^{\circ}$. In seinen Lösungen erzeugt Silbernitrat einen weissen, in Ammoniak schwer löslichen flockigen Niederschlag, Kupferacetat eine Trübung, Brom eine starke Fällung. Das Phenylosotriazolazimid ist beständig gegen kaltes übermangansaures Kali und reducirt ammoniakalische Silberlösung auch beim Erwärmen nicht. Durch kalte verdünnte Mineralsäuren wird es nicht verändert. Ebenso tritt beim Einleiten von trocknem Chlorwasserstoff in die ätherische Lösung eine Sprengung des Azimidringes nicht ein. Kocht man aber das Azimid mit Schwefelsäure von 50 pC. oder Salzsäure, so ist sofort Diazoverbindung nachweisbar. Sehr glatt erfolgt die Aufspaltung durch Acetylchlorid, wobei die Reaction nach folgender Gleichung verläuft: 
Thiele u. Schleussner, Diamidophenylosotriazol.

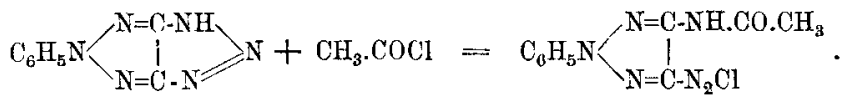

Das entstehende Acetylamidodiazophenylosotriazolchlorid wurde durch Eingiessen des Reactionsproductes in Aether isolirt und durch Ueberführung in Phenylosotriazolacetylazimid identificirt. Eisessig vermag das Azimid nur langsam zu spalten. Daher kuppelt dasselbe mit Dimethylanilin und Eisessig beim Kochen nur schwach.

$$
\text { Phenylosotriazolacetylazimid, }
$$

Die leichte Spaltbarkeit des Phenylosotriazolazimids durch Säuren macht die directe Acetylirung durch Behandlung mit Acetylchlorid unmöglich. Man muss daher von dem Monoacetyldiamidophenylosotriazol ausgehen, indem man dieses zunächst in die entsprechende Diazoverbindung überführt, aus deren Lösung durch Zusatz von Natriumacetat das Phenylosotriazolacetylazimid quantitativ ausfällt.

$10 \mathrm{~g}$ Monoacetyldiamidophenylosotriazol werden in $30 \mathrm{ccm}$ Eisessig gelöst und in eine stark gekühlte Lösung von $7 \mathrm{~g}$ Natriumnitrit und $15 \mathrm{~g}$ concentrirter Schwefelsäure in $300 \mathrm{ccm}$ Wasser eingetropft. In der gelben, meist etwas trüben Lösung ist jetzt das Sulfat des Acetylamidodiazophenylosotriazols enthalten. Tropft man dieselbe selbst in eine mässig ätzalkalische Lösung von R-Salz, so tritt keine Kuppelung ein, weil die Verbindung schneller als sie kuppelt in die Isoform umgelagert wird. Mit soda-alkalischem R-Salz erzielt man hingegen eine normale Farbstoffbildung.

Zur Umwandlung in das Acetylazimid wird die Diazolösung filtrirt und sofort mit einer concentrirten Lösung von $80 \mathrm{~g}$ Natriumacetat in Wasser versetzt, der entstehende Niederschlag abfiltrirt, mit viel Wasser gewaschen und scharf abgesaugt. Die letzten Spuren von Wasser werden durch Alkohol und 
Ligroïn entfernt und das farblose oder schwach gelb gefärbte Product an der Luft getrocknet. Es rerpufit bei $160^{\circ}$.

$0,2164 \mathrm{~g}$ gaben $0,4186 \mathrm{CO}_{2}$, entsprechend $0,11419 \mathrm{C}$, und 0,0706 $\mathrm{H}_{2} \mathrm{O}$, entsprechend $0,00784 \mathrm{H}$.

$0,1252 \mathrm{~g}$ gaben $42 \mathrm{~cm}$ Stickgas bei $14^{\circ}$ und $713 \mathrm{~mm}$ Druck.

$\begin{array}{ccr} & \text { Berechnet fïr } & \text { Gefunden } \\ & \mathrm{C}_{\mathbf{1 0}} \mathrm{N}_{6} \mathrm{I}_{8} \mathrm{O} & \\ \mathrm{C} & 52,63 & 52,75 \\ \mathrm{H} & \mathbf{3}, 50 & 3,62 \\ \mathrm{~N} & 36,84 & 36,99\end{array}$

Die Bestimmung des Molekulargewichts durch Gefrierpunktserniedrigung ergab das der obigen Formel entsprechende einfache Molekulargewicht.

Molekulargewichtsbestimmung in Nitrabenzol.

$0,1900 \mathrm{~g}$ ergaben eine Depression von $0,305^{\circ}$. Angewandtes Lösungsmittel $=20 \mathrm{~g}$.

$\begin{array}{ccc} & \text { Berechnet für } & \text { Gefunden } \\ & \mathrm{C}_{10} \mathrm{~N}_{6} \mathrm{H}_{8} \mathrm{O} & \\ \text { II } & 228 & 219,5\end{array}$

Die Vermuthung, dass das Phenylosotriazolacetylazimid überhaupt nicht nach dem Typus der Azimide zusammengesetzt, sondern eine aus mehreren Molekülen gebildete Diazoamidoverbindung sei, etwa

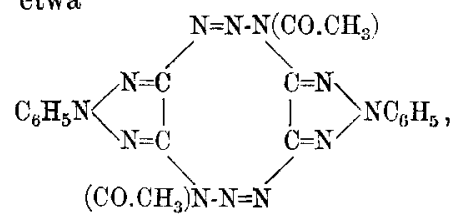

ist somit nicht bestätigt worden. $\mathrm{Zu}$ einer derartigen Vermuthung schien das auffallende Verhalten des Phenylosotriazolacetylazimids, das mehr die Eigenschaften einer Diazoamidoverbindung als die eines Azimids zeigt, zu berechtigen.

Während das Phenylosotriazolazimid durch Mineralsäuren verbaltnissmässig schwicrig gespalten wird, tritt bei dem Phenylosotriazolacetylazimid die Sprengung schon beim Kochen mit Essigsäure ein. Mit Dimethylanilin und Eisessig kuppelt es schon in der Kälte. 
Beim Erwärmen einer Lösung in Benzol oder Aether mit Acetylchlorid oder beim Einleiten von Chlorwasserstoff erfolgt Ausscheidung von

Acetylamidodiazophenylosotriazolchlorid,<smiles>COC(=O)Nc1nc2nc(n1)N=C2</smiles>

Dasselbe ist wenig beständig und färbt sich an der Luft unter Salzsäureverlust rasch gelb. Frisch gefällt ist es in Wasser leicht und klar löslich. Die Lösung trübt sich bei Abwesenheit von Mineralsäure schnell und verliert allmählich die Fähigkeit zu kuppeln unter Uebergang in Phenylosotriazolacetylazimid. Schmelzp. 150-1550 (Zersetzung).

$0,1703 \mathrm{~g}$ gaben $0,0899 \mathrm{AgCl}$, entsprechend 0,0222 $\mathrm{Cl}$.

$0,1280 \mathrm{~g}, \quad 36,6 \mathrm{ccm}$ Stickgas bei $16^{\circ}$ und $717 \mathrm{~mm}$ Druck.

$\begin{array}{ccc} & \text { Berechnet für } & \text { Gefunden } \\ & \mathrm{C}_{10} \mathrm{H}_{9} \mathrm{~N}_{6} \mathrm{NCT} & \\ \mathrm{Cl} & 13,42 & 13,05 \\ \mathrm{~N} & 31,75 & 31,42\end{array}$

Erhitzt man Phenylosotriazolacetylazimid mit Natronlauge, so wird die Acetylgruppe unter Bildung von Phenylosotriazolazimid abgespalten.

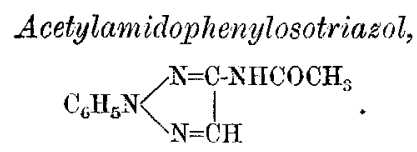

Dasselbe entsteht unter Entweichen von Aldehyd und Stickgas offenbar aus einer intermediär gebildeten Diazoverbindung beim Kochen des Acetylazimids mit Alkohol. Farblose, in Alkohol, Aether oder siedendem Benzol leicht, in Wasser schwer lösliche Nadeln. Schmelzp. $166^{\circ}$.

$0,1086 \mathrm{~g}$ gaben $27,6 \mathrm{~cm}$ Stickgas bei $17^{\circ}$ und $716 \mathrm{~mm}$ Druck.

$\begin{array}{ccc} & \text { Berechnet für } & \text { Gefunden } \\ & \mathrm{C}_{\mathbf{1 0}} \mathrm{H}_{10} \mathrm{~N}_{4} \mathrm{O} & \\ \mathrm{N} & 27,72 & 27,75\end{array}$




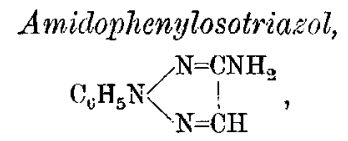

wird aus seinem Acetylderivat leicht durch Behandlung mit verdünnter Natronlauge erhalten.

Man kocht Phenylosotriazolacetylazimid mit dem 15- bis 20 fachen Gewicht absoluten Alkohols, bis alles Azimid vollständig zersetzt und die Lösung klar geworden ist. Die erhaltene Lösung von Acetylamidophenylosotriazol dampft man zur Trockne ein und erwärmt den Rückstand $1 / 2$ Stunde mit Natronlauge auf dem Wasserbade. Das sich ausscheidende Oel wird mit Aether aufgenommen, die ätherische Lösung mit Wasser gewaschen, der Aether abdestillirt und der schwach braun gefärbte Rückstand wiederholt aus heissem Wasser umkrystallisirt. Farblose, lange Nädelchen vom Schmelzp. $70^{\circ}$, leicht löslich in Alkohol, Benzol, Chloroform, schwer losslich in kaltem, leichter in heissem Wasser.

$0,1769 \mathrm{~g}$ gaben $0,3908 \mathrm{CO}_{2}$, entsprechend $0,10658 \mathrm{C}$, und $0,0836 \mathrm{H}_{2} \mathrm{O}$, entsprechend $0,0093 \mathbf{H}$.

$0,1056 \mathrm{~g}$ gaben $33,8 \mathrm{ccm}$ Stickgas bei $18^{\circ}$ und $722,5 \mathrm{~mm}$ Druck.

Berechnet füir Gefunden

$\begin{array}{lcr} & \mathrm{C}_{8} \mathrm{H}_{8} \mathrm{~N}_{4} & 60,23 \\ \mathrm{C} & 60,00 & 5,25 \\ \mathrm{H} & 5,00 & 55,10 \\ \mathrm{~N} & 35,00 & 35,10\end{array}$

Das Amidophenylosotriazol bildet mit Mineralsäuren und Essigsäure leicht lösliche Salze. Es reducirt alkalische Silberlösung nicht oder nur sehr schwach beim Erwärmen. Mit Eisenchlorid färbt es sich schwach blau (vielleicht in Folge geringer Verunreinigung mit Diamidophenylosotriazol). Die Amidogruppe ist diazotirbar; die entsprechende Diazoverbindung kuppelt mit R-Salz zu einem orangerothen Azofarbstoffe. In Lösungen des Amidophenylosotriazols verursacht alkalische Bromlösung keine Gasentwickelung, Benzaldehyd keine Fällung.

Aus $5 \mathrm{~g}$ Phenylosotriazolacetylazimid, bezw. $4 \mathrm{~g}$ Acetylamidophenylosotriazol wurde $1 \mathrm{~g}$ reines Amidophenylosotriazol erhalten. 


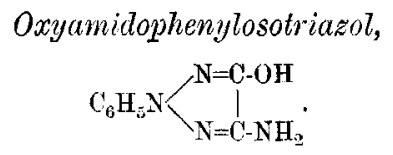

entsteht als Nebenproduct bei der Condensation des Oxalenphenylbydrazidamidoxims. Es ist in der bei der Darstellung des Diamidophenylosotriazols zurückbleibenden alkalischen Lösung in wechselnden Mengen (circa 3 pC.) enthalten. Diese Lösung wird mit Salzsäure schwach angesäuert und wiederholt ausgeäthert. Die ätherischen, gelb gefärbten Lösungen werden vereinigt und der Aether abdestillirt. Der Rückstand besteht grösstentheils aus Oxyamidophenylosotriazol. Dasselbe ist leicht löslich in Alkohol, Benzol, Aceton, Chloroform, sehr schwer in Wasser; durch wiederholtes Umkrystallisiren und Behandlung der Lösungen mit Thierkohle wird das Amin in farblosen, langen Nadeln erhalten, die bei $181^{\circ}$ unter Zersetzung schmelzen.

$0,2329 \mathrm{~g}$ gaben $0,4665 \mathrm{CO}_{2}$, entsprechend $0,12723 \mathrm{C}$, und $0,1013 \mathrm{H}_{2} \mathrm{O}$, entsprechend $0,0112 \% \mathrm{H}$.

$0,1941 \mathrm{~g}$ gaben $55,6 \mathrm{~cm}$ Stickgas bei $10^{a}$ und $715 \mathrm{~mm}$ Druck.

$\begin{array}{ccr} & \text { Berechnet fïr } & \text { Gefinden } \\ & \mathrm{C}_{5} \mathrm{I}_{8} \mathrm{~N}_{4} \mathrm{O} & \\ \mathrm{C} & 54,54 & 54,62 \\ \mathrm{H} & 4,54 & 4,83 \\ \mathrm{~N} & 31,81 & 32,19\end{array}$

Das Oxyamidophenylosotriazol löst sich schwer in verdünnten Säuren, leicht in Alkalien, kohlensauren Alkalien und Ammoniak. Die wässrige Lösung reagirt sauer und färbt beim Erwärmen einen Fichtenspahn intensiv rothgelb. Analog dem Diamidophenylosotriazol giebt es mit Eisenchlorid eine äusserst scharfe und empfindliche Farbenreaction. Der entstehende, prachtvoll violette, in Aether, Alkohol oder Chloroform leicht lösliche Farbstoff wird durch Säuren zerstört, durch Alkalien oder Ammoniak tiefblau.

Brom und Natronlauge bewirken in Lösungen des Oxyamidophenylosotriazols eine intensiv carmoisinrothe Färbung, Benzaldehyd einen gelben Niederschlag von 
Benzaloxyamidophenylosotriazol,

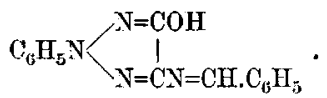

Dasselbe ist durch Fällung nur schwer krystallisirt zu erhaiten. Leicht gelingt die Condensation, wenn man molekulare Mengen von Oxyamidophenylosotriazol und Benzaldehyd mit wenig Eisessig erhitzt. Die beim Erkalten sich ausscheidenden schwefelgelben Krystalle werden aus Eisessig oder verdünntem Alkohol umkrystallisirt. Sie sind löslich in verdünnter Natronlauge, kohlensauren Alkalien oder Ammoniak, unlöslich in Essigsäure, nicht ganz klar löslich in Mineralsäuren. Schmelzpunkt $173^{\circ}$.

$0,2389 \mathrm{~g}$ gaben $0,5949 \mathrm{CO}_{2}$, entsprechend $0,16224 \mathrm{C}$, und $0,1009 \mathrm{H}_{2} \mathrm{O}$, entsprechend $0,01121 \mathrm{H}$.

$0,1014 \mathrm{~g}$ gaben $19,4 \mathrm{cem}$ Stickgas bei $11^{0}$ und $716 \mathrm{~mm}$ Druck.

Berechnet für Gefunden

$\begin{array}{ccr} & \mathrm{C}_{15} \mathrm{H}_{12} \mathrm{~N}_{4} \mathrm{O} & \\ \mathrm{C} & 68,18 & 67,91 \\ \mathrm{H} & 4,04 & 4,69 \\ \mathrm{~N} & 21,21 & 21,26\end{array}$

Diazooxyphenylosotriazol, $\mathrm{C}_{6} \mathrm{H}_{5} \mathrm{~N}\left\langle\underset{\mathrm{N}=\mathrm{C}-\mathrm{OH}}{\mathrm{N}=\mathrm{C}-\mathrm{N}_{2} \mathrm{OH}}\right.$

Durch Einwirkung von salpetriger Säure auf das Oxyamidophenylosotriazol wird, wie zu erwarten ist, die Amidogruppe diazotirt. Man verfährt am besten wie bei der Diazotirung des Diamins: 2 g Oxyamidophenylosotriazol werden in circa $20 \mathrm{ccm}$ Alkohol gelöst, die Lösung mit Wasser versetzt, bis Trübung erfolgt, und langsam unter Umrühren in cine stark gekühlte Lösung von $1,6 \mathrm{~g}$ Natriumnitrit in $180 \mathrm{ccm}$ Wasser und $20 \mathrm{ccm}$ Salzsäure eingetropft. Dic dunkel gefärbte Diazolösung wird sofort filtrirt. Sie ist etwas weniger beständig als die des Diamins, indem sie schon nach vier Tagen die Fähigkeit mit R-Salz zu kuppeln, nahezu verliert und bei längerem Erwärmen ohne Gasentwickelung vollständig zersetzt wird. Das 
Diazooxyphenylosotriazol lagert sich ebenfalls leicht in die entsprechende Isodiazoverbindung um.

Beim Behandeln der schwefelsauren IDiazolösung mit feiı vertheiltem metallischem Kupfer entweicht schon in der Kälte Stickstoff. Der sauren Lösung kanı nach Beendigung der Reaction durch Aether ein Körper entzogen werden, der aus heissem Wasser in farblosen, langen Nadeln krystallisirt. Derselbe schmilzt bei $124^{\circ}$, löst sich leicht in Alkohol, Chloroform oder Benzol und reducirt alkalische Silberlösung nicht. Die Stickstoffbestimmung ergab einen Stickstoffgehalt von $26,02 p \mathrm{C}$. was der Formel eines Monoxyphenylosotriazols

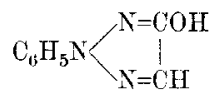

entsprechen würde. Es hat also wohl der in der Diazolösung enthaltene Alkohol reducirend gewirkt, da andererscits eine Dioxyverbindung zu erwarten gewesen wäre.

$0,1880 \mathrm{~g}^{\circ}$ gaben $44 \mathrm{cem}$ stickgas bei $14^{0}$ und $719 \mathrm{~mm}$ Druck.

$$
\begin{array}{ccc} 
& \text { Berechnet für } & \text { Gefunden } \\
& \mathrm{C}_{8} \mathrm{H}_{2} \mathrm{~N}_{3} \mathrm{O} & \\
\mathrm{N} & 26,08 & 26,02
\end{array}
$$

Weitere Untersuchungen sollen diese Vermuthung bestätigen.

$$
\begin{aligned}
& \beta-\text { Naphtylaminazooxyphenylosotriazol, } \\
& \qquad \begin{array}{l}
\mathrm{C}=\mathrm{C} \cdot \mathrm{N}=\mathrm{N}\left(\mathrm{C}_{\mathbf{1 0}} \mathrm{H}_{0}\right) \mathrm{HH}_{2} \mathrm{~N}, \\
\mathrm{~N}=\mathrm{C}-\mathrm{OH}
\end{array}
\end{aligned}
$$

wird durch Kuppeln von Diazooxyphenylosotriazol mit $\beta$-Naphtylamin erhalten. Braunroth, in Ammoniak mit rosenrother, in concentrirter Schwefelsäure mit indigblauer Farbe löslich.

$0,0788 \mathrm{~g}$ gaben $18,4 \mathrm{ccm}$ Stickgas bei $14^{0}$ und $707 \mathrm{~mm}$ Druck.

$$
\begin{array}{ccc} 
& \text { Berechnet für } & \text { Gefunden } \\
& \mathrm{C}_{18} \mathrm{~N}_{6} \mathrm{H}_{14} \mathrm{O} & \\
\mathrm{N} & 25,4 \tilde{5} & 2 \tilde{5}, 32
\end{array}
$$




\section{Einwirkung von Cyan auf Amidoguanidin und Semicarbazid.}

Cyan und Amidoguanidin (Cyansemicarbazid),

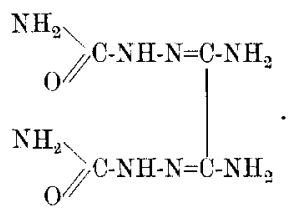

Cyan wird nur von freiem Amidoguanidin, nicht von Amidoguanidinsalzen absorbirt. Lösungen der freien Base wurden durch Versetzen des Nitrats mit der berechneten Menge Kalilauge in der Kälte dargestellt. Sie färben sich beim Einleiten von Cyan unter Erwärmen gelb und trüben sich nach einiger Zeit unter Abscheidung eines mehr oder weniger gefärbten Niederschlags. Die Absorption des Gases erfolgt nur langsam. Gleichzeitig verlaufende Nebenreactionen, besonders aber die leichte Löslichkeit und Unbeständigkeit der entstehenden Cyanverbindungen, erschweren die Reinigung und Trennung der verschiedenen Reactionsproducte. Es konnten zwar einige hochschmelzende Körper isolirt werden, indessen wurde von einer eingehenderen Untersuchung Abstand genommen, da sio nur in ungenügender Ausbeute erhalten wurden.

Etwas bessere Resultate wurden durch Einwirkung alkoholischer Cyanlösungen auf Amidoguanidin erzielt.

Leitet man Cyan in stark abgekühlten Alkohol, so gelingt es leicht, fünf- bis sechsprocentige Lösungen darzustellen. Trägt man in eine derartige Lösung ( $300 \mathrm{~g}$ enthaltend $16 \mathrm{~g}$ Cyan) eine concentrirte IJösung von Amidoguanidinnitrat (30 $\mathrm{g}$ in $60 \mathrm{~g}$ Wasser) ein und versetzt mit der berechneten Menge alkoholischem Kali, so verschwindet der Geruch des Cyans fast vollständig. Die vom Salpeter abfiltrirte Lösung hinterlässt, nach vorsichtigem Ansäuern mit verdünnter Salpetersäure, beim Eindampfen neben beträchtlichen Mengen von salpetersaurem Ammoniak einen Körper von der Zusammensetzung $\mathrm{C}_{4} \mathrm{~N}_{10} \mathrm{I}_{12} \mathrm{O}_{8}$. Derselbe wird nach wiederholtem Umkrystallisiren aus Wasser 
in farblosen Nadeln von constantem Schmelzp. $223^{\circ}$ (unter Zersetzung) erhalten.

$0,1738 \mathrm{~g}$ gaben $0,0950 \mathrm{CO}_{2}$, entsprechend $0,0259 \mathrm{C}$, und $0,0649 \mathrm{H}_{2} \mathrm{O}$, entsprechend $0,0072 \mathrm{H}$.

$0,1052 \mathrm{~g}$ gaben $41,6 \mathrm{cem}$ Stickgas bei $20^{0}$ und $722 \mathrm{~mm}$ Druck.

$\begin{array}{ccr} & \text { Berechnet für } & \text { 'icfiunden } \\ & \mathrm{C}_{4} \mathrm{~N}_{10} \mathrm{H}_{12} \mathrm{O}_{\mathrm{s}} & \\ \mathrm{C} & 14,63 & 14,90 \\ \mathrm{H} & 3,66 & 4,14 \\ \mathrm{~N} & 42,68 & 42,81 .\end{array}$

Die wässrige Lösung dieses Körpers reagirt sauer und giebt mit Diphenylamin oder Ferrosulfat und concentrirter Schwefelsäure die charakteristischen Reactionen auf Salpetersäure. Durch Aetzalkalien wird Ammoniak, durch Brom sowohl in saurer als auch alkalischer Lösung Stickstoff abgespalten. Ammoniakalische Silberlösung wird bei gelindem Erwärmen sofort reducirt. Dieses Verhalten und das reichliche Auftreten von Ammoniak beim Eindampfen der cyanhaltigen Amidoguanidinlösung (neben freiem Cyan, Cyanwasserstoff und anderen Zersetzungsproducten) lassen den Schluss nicht unberechtigt erscheinen, dass intermediär Cyanamidoguanidin gebildet wurde, das dann in Cyansemicarbazid, beziehungsweise dessen Nitrat, überging:

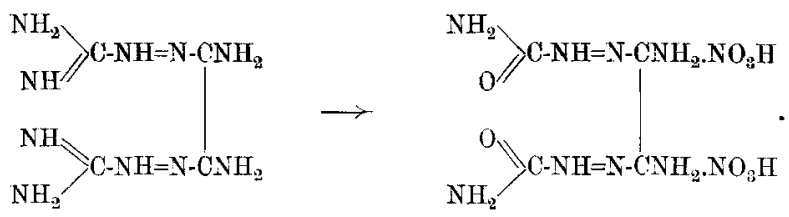

Für die Condensation zu einem Derivat des Osotriazols war natürlich eine derartige Verbindung nicht geeignet.

Cyan und Senicarbazid.

Dicyansemicarbazid,

$\mathrm{NH}_{2} \cdot \mathrm{CO}-\mathrm{NHN}=\underset{\mathrm{CN}}{\mathrm{C}}-\mathrm{NH}_{2}$.

$50 \mathrm{~g}$ salzsaures Semicarbazid werden in $250 \mathrm{ccm}$ Wasser gelöst, stark abgekühlt und mit etwas mehr als der berechneten 
Menge Aetzkali (26 g) versetzt. In die alkalische Lösung wird ein nicht $\mathrm{zu}$ schneller Strom von Cyangas eingeleitet. Die Absorption erfolgt sehr langsam und träge, sofern sie nicht durch beständiges, krüftiges Schüttteln und Kühlung befördert wird ${ }^{10}$ ). Die Flüssigkeit verändert sich anfangs nicht. Nach einiger Zeit trübt sie sich unter Abscheidung eines weissen, ziemlich compacten Niederschlags. Von Zeit zu Zeit wird die Lösung auf ihre Reaction geprüft und eventuell durch Zusatz von wenig Aetzkali wieder alkalisch gemacht. Wenn kein Gas mehr absorbirt wird, wird das Einleiten unterbrochen, der Niederschlag nach $1 / 4$ Stunde abgesaugt, mit wenig Fiswasser gewaschen und aus heissem Wasser umkrystallisirt. Die Ausbeute schwankt zwischen 30 und $40 \mathrm{~g}$, das heisst 52 bis $70 \mathrm{pC}$. der Theorie. Das unveränderte Semicarbazid kann aus dem Filtrat leicht als Acetonverbindung wiedergewonnen werden.

Das Dicyansemicarbazid krystallisirt aus der heissen, wässrigen Lösung in farblosen, büschelförmigen Nadeln, die nicht schmelzen, sondern unter Entwickelung von Ammoniak, Cyanwasserstoff und anderen Zersetzungsproducten langsam verkohlen. Es reducirt alkalische Silberlösung stark und giebt mit Kupferacetat einen schmutzig grünen Niederschlag. In Alkalien und kohlensauren Alkalien ist es mit gelber Farbe unter theilweiser Zersetzung löslich. Mineralsäuren lösen das Dicyansemicarbazid gleichfalls auf, Essigsäure wirkt in der Kälte nicht ein.

10) Um die Absorption des Cyans zu befördern, bedient man sich zweckmässig einer Vorrichtung, die anch in anderen Fiallen von Vortheil sein dürfte. Die zn süttigende Flüssigkeit befindet sich in einem weithalsigen, mit Kork verschliessbaren (alase, durch dessen Stopfen ein Witt'scher Rüihrer hindurchgeht, welcher als Nührung ein in die Flüssigkeit eintauchendes Glasrohr hat. Das Cyan wird unter den Rührer geleitet, so das es durch die Bewegung desselben angesaugt und in feinster Vertheilung durch die Flüssigkeit getrieben wird. Etwa abriehende Gase werden durch eine Waschtlasche geleitet, an welcher man verfolgen kam, ob alles eingeleitete Gas absorbirt wird. Bei nicht zu stümischom Einleiten des Gases ist die Absorption vollstrindig. 
$0,1992 \mathrm{~g}$ gaben $0,2060 \mathrm{CO}_{\mathrm{a}}$, entsprechend $0,05617 \mathrm{C}$, und $0,0728 \mathrm{H}_{2} \mathrm{O}$, entsprechend $0,0080 \mathrm{H}$.

$0,1210 \mathrm{~g}$ gaben $\breve{9} 9,4 \mathrm{ccm}$ Stickgas bei $10^{\circ}$ und $712 \mathrm{~mm}$ Druck.

$\begin{array}{ccr} & \text { Berechnet für } & \text { Gefunden } \\ & \mathrm{C}_{3} \mathrm{~N}_{5} \mathrm{H}_{5} \mathrm{O} & \\ \mathrm{C} & 28,34 & 28,19 \\ \mathrm{H} & 3,93 & 4,06 \\ \mathrm{~N} & 55,11 & 55,02\end{array}$

Amidoxim des Dicyansemicarbazids,<smiles>N=C(C(N)=O)C(N)=[18O]</smiles>

Dasselbe entsteht leicht und quantitativ, wenn man eine wässrige Lösung von Dicyansemicarbazid (10 g in $300 \mathrm{ccm}$ heissem Wasser) mit der äquivalenten Menge Hydroxylamin (aus dem Chlorhydrat mit Soda bereitet) versetzt und zur Vollendung der Reaction einige Minuten auf dem Wasserbade erwärmt. Beim Erkalten scheiden sich feine, gelblichweisse Nädelchen aus, die durch wiederholtes Umkrystallisiren aus heissem Wasser farblos werden. Ihre Zusammensetznng entspricht der Formel $\mathrm{C}_{3} \mathrm{H}_{8} \mathrm{~N}_{6} \mathrm{O}_{2}$.

$0,1742 \mathrm{~g}$ gaben $0,1428 \mathrm{CO}_{2}$, entsprechend $0,03894 \mathrm{C}$, und $0,0812 \mathrm{H}_{2} \mathrm{O}$, entsprechend $0,00902 \mathrm{H}$.

$0,0938 \mathrm{~g}$ lieferten 44,6 ccm Stickgas bei $12^{\circ}$ und $711 \mathrm{~mm}$ Druck.

$\begin{array}{ccr} & \text { Berechnet für } & \text { Gefunden } \\ & \mathrm{C}_{3} \mathrm{H}_{8} \mathrm{~N}_{6} \mathrm{O}_{2} & \\ \mathrm{C} & 22,50 & 22,35 \\ \mathrm{HI} & 5,00 & 5,17 \\ \mathrm{~N} & 52,50 & 52,75\end{array}$

Das Amidoxim des Dicyansemicarbazids unterscheidet sich in seinem Aussehen und in seinen physikalischen Eigensehaften von dem Dicyansemicarbazid nur wenig. Es zeigt die gleichen Löslichkeitsverhältnisse, dasselbe Verhalten gegen verdünnte Säuren und Alkalien und hat ebenfalls keinen Schmelzpunkt, sondern verkohlt bei höherer Temperatur.

Ein wesentlicher Unterschied besteht in dem verschiedenen Verhalten gegen Eisenchlorid. Während Lösungen des Dicyan- 
semicarbazids sich auf Zusatz von Eisenchlorid nur schwach gelb und beim Erwärmen braun färben, bewirken Spuren des Oxims in verdünnten Eisenchloridlösungen eine dunkel röthlichbraune Färbung, die beim Erwärmen verschwindet.

Versuche, das Amidoxim durch Erhitzen mit Wasser zu einem Diamidoosotriazol zu condensiren:

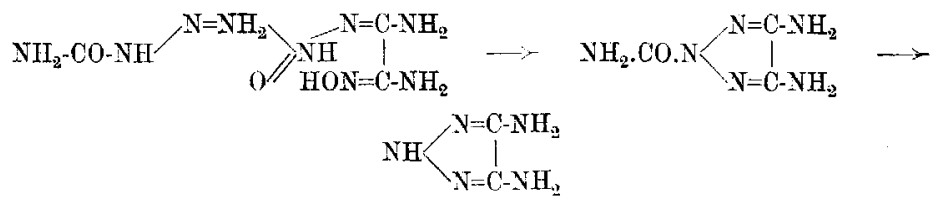

führten statt dessen zu zwei Körpern von den Formeln $\mathrm{C}_{3} \mathrm{H}_{5} \mathrm{~N}_{5} \mathrm{O}_{2}$ (aus dem Amidoxim durch Austritt eines Moleküls Ammoniak entstehend) und $\mathrm{C}_{6} \mathrm{H}_{14} \mathrm{~N}_{8} \mathrm{O}_{7}$. Setzt man Soda als Condensationsmittel $\mathrm{zu}$, so tritt weitere Zersetzung unter Bildung ron Hydrazin und Oxalsäure ein. Die Constitution dieser Verbindungen ist zur Zeit noch unaufgeklärt.

Die Verbindung $\mathrm{C}_{3} \mathrm{H}_{5} \mathrm{~N}_{5} \mathrm{O}_{z}$ entsteht als weisser Niederschlag, wenn man die zwei Stunden mit der zehnfachen Menge Wasser auf $160^{\circ}$ erhitzte Oximlösung mit Essigsäure ansäuert. Leicht löslich in Ammoniak, Alkalien, kohlensauren Alkalien, Mineralsäuren; schwer löslich in Wasser, unlöslich in Essigsäure. Zur Analyse wurde dieses Condensationsproduct durch wiederholtes Auflösen in Ammoniak und Fällen mit Essigsäure gereinigt, aus heissem Wasser umkrystallisirt und im Toluolbade bis zur Gewichtsconstanz getrocknet.

I. $0,1910 \mathrm{~g}$ saben $0,1749 \mathrm{CO}_{\mathrm{g}}$, entsprechend $0,0477 \mathrm{C}$, und 0,0618 $\mathrm{H}_{2} \mathrm{O}$, entsprechend $0,00686 \mathrm{H}$.

$0,0891 \mathrm{~g}$ gaben $38,3 \mathrm{ccm}$ Stickgas bei $10^{\circ}$ und $723 \mathrm{~mm}$ Druck.

II. $0,1725 \mathrm{~g} \quad$ " $0,1600 \mathrm{CO}_{2}$, entsprechend $0,04367 \mathrm{C}$, und 0,0520

$\mathrm{H}_{\mathrm{g}} \mathrm{O}$, entsprechend $0,00578 \mathrm{H}$.

$0,0859 \mathrm{~g}$ gaben $38,6 \mathrm{ccm}$ Stickgas bei $18,5^{\circ}$ und $716 \mathrm{~mm}$ Druck.

\begin{tabular}{ccrr} 
& Berechnet für & \multicolumn{2}{c}{ Gefunden } \\
& $\mathrm{C}_{3} \mathrm{H}_{5} \mathrm{~N}_{5} \mathrm{O}_{2}$ & I. & II. \\
() & 25,17 & 24,97 & 25,29 \\
$\mathrm{H}$ & $\$, 49$ & 3,59 & 3,34 \\
$\mathrm{~N}$ & 48,95 & 48,93 & 48,75
\end{tabular}


Die aus wässriger Lösung erhaltenen Krystalle sind farblos, unschmelzbar und unlöslich in den gebräuchlichen organischen Lösungsmitteln. Die Lösung reagirt schwach sauer und giebt mit Kupferacetat einen amorphen, grünlichen Niederschlag. Das Silbersalz ist weiss und sehr schwer löslich in Ammoniak. Eisenchloridlösung wird intensiv rothbraun gefärbt, ammoniakalische Silberlösung bei längerem Erwärmen reducirt. Beim Erhitzen mit Soda auf $180^{\circ}$ tritt nur geringe Zersetzung ein; Salzsäure spaltet bei dieser Temperatur Hydrazin ab.

Die Verbindung $\mathrm{C}_{6} \mathrm{H}_{14} \mathrm{~N}_{8} \mathrm{O}_{7}$ ist nach dem Ausfällen des ersten Körpers neben essigsaurem Ammoniak der Hauptbestandtheil der zurückbleibenden Lösung. Dieselbe wird soweit eingedampft, bis Ausscheidung von Krystallen beginnt, dann mit dem 30 fachen Volumen absolutem Alkohol versetzt, die ausgeschiedenen Krystalle abgesaugt, in wenig Wasser gelöst und nochmals mit Alkohol gefällt. Man erhält auf diese Weise weisse, seidenglänzende, unschmelzbare Nadeln, deren wässrige, essigsaure Lösung eine schön rosenrothe Farbe annimmt, wenn man Spuren von Brom zusetzt. Die rothe Farbe wird durch überschüssiges Brom oder Mineralsäuren zum Verschwinden gebracht. Bei Anwendung von schwefliger Säure kehrt sie bei vorsichtigem Zusatz von Brom wieder.

$0,2028 \mathrm{~g}$ gaben $0,1742 \mathrm{CO}_{2}$, entsprechend $0,0475 \mathrm{C}$, und $0,08448 \mathrm{H}_{3} \mathrm{O}$, entsprechend $0,0094 \mathrm{H}$.

$0,1049 \mathrm{~g}$ gaben $33,1 \mathrm{cem}$ Stickgas bei $12^{0}$ und $731 \mathrm{~mm}$ Druck.

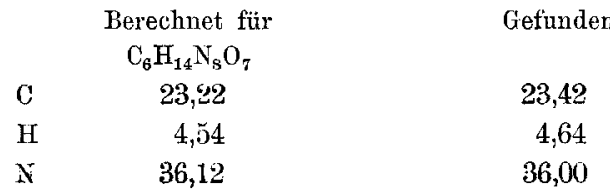

Die Verbindung $\mathrm{C}_{6} \mathrm{H}_{14} \mathrm{~N}_{8} \mathrm{O}_{7}$ giebt mit unterbromigsaurem Natrium starke Stickstoffentwickelung, mit concentrirter Schwefelsäure neben Stickstoff hauptsächlich Kohlenoxyd. Sie entfärbt Permanganat sofort und wirkt auf Silberlösungen stark reducirend. Beim Erhitzen mit kohlensaurem Alkali oder Mineral- 
Thiele u. Schleussner, Diamidophenylosotriazol. 167 säuren auf $140-150^{\circ}$ werden beträchtliche Mengen Kohlensäure und Hydrazin abgespalten.

\section{Einige Derivate der Phenylhydrazinoxalsäure.}

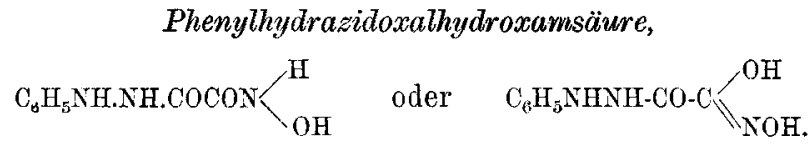

Dieselbe entsteht leicht durch Einwirkung von Hydroxylamin auf Phenylhydrazinoxalester ${ }^{11}$ ), und zwar ist die Anwendung von zwei Molekülen Hydroxylamin vortheilhaft.

$140 \mathrm{~g}$ Hydroxylaminchlorhydrat werden in $600 \mathrm{ccm}$ Methylalkohol heiss gelöst und mit einer Lösung von $46 \mathrm{~g}$ Natrium in $600 \mathrm{ccm}$ Alkohol vermischt. Die vom Kochsalz abfiltrirte Hydroxylaminlösung wird noch etwas warm in eine heisse alkoholische Lösung von $200 \mathrm{~g}$ Phenylhydrazidoxalester eingegossen. Nach kurzer Zeit beginnt die Ausscheidung der in Alkohol sebr schwer löslichen Hydroxamsäure in farblosen Blättchen, welche mit Alkohol ausgewaschen werden. Ausbeute $82 \mathrm{~g}$ rohe Säure. Zur weiteren Verarbeitung ist das Präparat genügend rein, zur Analyse wurde es zuerst aus siedendem Wasser unter Zusatz von wenig Essigsäure, dann nochmals aus Methylalkohol umkrystallisirt. Farblose, bei $172^{0}$ unter Zersetzung schmelzende Krystalle.

I. $0,1809 \mathrm{~g}$ gaben $0,3264 \mathrm{CO}_{2}$, entsprechend $0,08902 \mathrm{C}$, und 0,0782 $\mathrm{H}_{2} \mathrm{O}$, entsprechend $0,00870 \mathrm{H}$.

$0,1622 \mathrm{~g}$ gaben $31,4 \mathrm{ccm}$ Stickgas bei $12,5^{\circ}$ und $71.7 \mathrm{~mm}$ Druck.

IT. $0,1703 \mathrm{~g} \quad, \quad 0,3086 \mathrm{CO}_{\Omega}$, entsprechend $0,08416 \mathrm{C}$, und 0,0728 $\mathrm{H}_{2} \mathrm{O}$, entsprechend $0,0081 \mathrm{H}$.

$0,2318 \mathrm{~g}$ gaben $45,0 \mathrm{~cm}$ Stickgas bei $16^{\circ}$ und $723 \mathrm{~mm}$ Druck.

11) Bülow, diese Annalen 286, 197. Die Darstellung des Fsters geschieht zweckmässig durch Stehenlassen ron Oxalester (250 g) mit $1.00 \mathrm{~g}$ Phenylhydrazin in $200 \mathrm{ccm}$ Alkohol. Die nach zwei Tagen zu einem Brei vou sehr kleinen, gelben Krystallen erstarte Flüssigkeit wird abgesangt und der mit wenig verdünntem Alkohol gewaschene Rückstand aus Alkohol (1000 ccm) umkrystallisirt. 


\begin{tabular}{ccrr} 
& Berechnet für & \multicolumn{1}{c}{ Gefunden } \\
$\mathrm{C}_{8} \mathrm{~N}_{3} \mathrm{H}_{9} \mathrm{O}_{3}$ & I. & II. \\
$\mathrm{C}$ & 49,23 & 49,20 & 49,42 \\
$\mathrm{H}$ & 4,61 & 4,80 & 4,74 \\
$\mathrm{~N}$ & 21,53 & 21,61 & 21,51
\end{tabular}

In den üblichen Lösungsmitteln sehr schwer löslich, leichter in beissem Wasser, leicht in alkalischen Flüssigkeiten und aus diesen Lösungen durch Essigsäure wieder fällbar. Durch dieses Umfällen steigt der Schmelzpunkt bis auf $178-180^{\circ}$, ohne dass damit eine Aenderung in Zusammensetzung und Eigenschaften verbunden wäre ${ }^{12}$ ). Eisenchlorid giebt mit der wässrigen Lösung die violettrothe Reaction der Hydroxamsäuren, ebenso giebt die Säure die Bülow'sche Reaction. Erhitzt man die Säure mit Alkohol im Einschlussrohre, so entsteht neben anderen Zersetzungsproducten (beim Oeffnen sehr starker Druck und Aufschäumen) Phenylhydrazidoxalsäureamid ${ }^{13}$ ). Ebensowenig gelang die Condensation zu einem Osotriazolderivat mit andercn Mitteln. Kochen mit Eisessig bewirkt totale Zersetzung unter Bildung geringer Mengen Phenylurazol, Kochen des Natriumsalzes in wässriger Lösung oder Kochen mit Soda führt zur Phenylhydrazinoxalsäure.

12) Tine ans dem Natriumsalz gefällte und bei $180^{\circ}$ schmelzende Säure (nochmals aus Wasser krystallisirt Schmelzp. 178') ergab bei der Analyse auf Hydroxamsäure stimmende Zahlen (gefunden C 49,22, berechnet 49,22; H 4,77, berechnet 4,62; N 21,95, berechnet 21,53 pc.). Analysen des aus der alkoholischen Jösung gefallenen, mit Alkohol und Aether gewaschenen Rohproductes ergaben Zahlen, die auf ein Gemisch der Säure mit ihrem Hydroxylaminsalz denten.

13) Das bisher noch nicht beschriebene Phenylhydrazidoxalsäureamid entsteht quantitativ, wenn man eine Lösung des Phenylhydrazidoxalesters mit Ammoniak versetzt. Sehr schwer lösliche, schwach gelbe, bei $230-233^{\circ}$ unter Zersetzung schmelzende Krystalle.

$\begin{array}{ccr} & \text { Berechnet für } & \text { Gefunden } \\ & \mathrm{C}_{8} \mathrm{~N}_{3} \mathrm{H}_{9} \mathrm{O}_{2} & \\ \mathrm{C} & 53,63 & 53,57 \\ \mathrm{H} & 5,02 & 5,10 \\ \mathrm{~N} & 23,16 & 23,25\end{array}$


Thiele u. Schleussner, Diamidophenylosotriazol. 169

Phenylhydrazidoxalacetylhydroxamsäure, $\mathrm{C}_{\mathrm{u}} \mathrm{H}_{5} \mathrm{NHINH} . \mathrm{CO} . \mathrm{C} \leqslant \mathrm{NOCOCH}_{3}$.

Die Hydroxamsäure wird unter Umrühren mit ihrem doppelten Gewicht Essigsäureanhydrid übergossen. Die Masse, welche sich unter starker Erwärmung gelb färbt, wird, sobald sie nicht mehr bröcklich, sondern halbflüssig ist, mit wenig Alkohol versetzt, scharf abgesaugt und mit Alkohol ausgewaschen. Lässt man die Masse ohne Alkoholzusatz stehen, so tritt leicht unter Auf kochen eine tiefgehende Zersetzung ein.

Das rohe Acetylderivat bildet ein gelbes Pulver, welches je nach der Reinheit der angewandten Hydroxamsäure zwischen $147-163^{\circ}$ schmilzt. Durch Umkrystallisiren aus Eisessig erhält man schwach gelbe, bei $147^{\circ}$ schmelzende Krystalle.

I. $0,1301 \mathrm{~g}$ gaben $0,2408 \mathrm{CO}_{2}$, entsprechend $0,06567 \mathrm{C}$, und $0,0572 \mathrm{H}_{2} \mathrm{O}$, entsprechend $0,00635 \mathrm{H}$.

$0,1844 \mathrm{~g}$ gaben $29,4 \mathrm{~cm}$ Stickgas bei $12^{0}$ und $719 \mathrm{~mm}$ Druek.

II. $0,1621 . \mathrm{g} \quad, 23,7 \mathrm{ccm}$ Stickgas $" 10^{\circ}, 715 \mathrm{~mm}$ Druck.

\begin{tabular}{ccrc} 
& Berechnet für & \multicolumn{2}{c}{ Gefunden } \\
$\mathrm{C}$ & $\mathrm{C}_{10} \mathrm{~N}_{3} \mathrm{H}_{11} \mathrm{O}_{4}$ & I. & II. \\
$\mathrm{C}$ & $\tilde{0} 0,62$ & 50,47 & - \\
$\mathrm{H}$ & 4,64 & 4,88 & - \\
$\mathrm{N}$ & 17,72 & 17,89 & 17,85
\end{tabular}

Kaum löslich in Wasser, schwer in heissem Alkohol, leicht in Carbonaten. Eisenchlorid giebt mit der Acethydroxamsäure keine Reaction, woraus man auf Acetylirung der Oximidogruppe schliessen kann. Beim Kochen mit Alkalien entsteht Phenylhydrazinoxalsäure, so dass also beim Acetyliren keine Umlagerung erfolgt ist. Eine Condensation des Acetylderivates unter Abspaltung von Essigsäure hätte zu dem gesuchten Phenyldioxyosotriazol führen sollen:

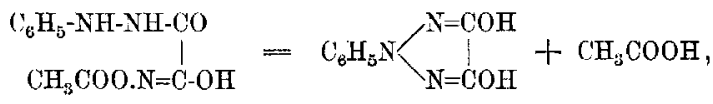

indessen konnte nur ein Condensationsmittel, das Dimethylanilin, gefunden werden, welches Essigsäure ohne totale Zerstörung 
des Moleküls abspaltete; das Condensationsproduct erwies sich als identisch mit dem Phenylurazol,<smiles>CCCC(=O)NC(=O)NC=O</smiles>

von Pinner ${ }^{14}$ ), so dass bei der Essigsäureabspaltung eine Umlagerung vor sich gegangen war.

\section{Phenylurazol aus Phenylhydrazidacethydroxamsäure.}

$25 \mathrm{~g}$ Hydroxamsäure werden in einem Literkolben mit dem sechs- bis achtfachen Gewicht Dimethylanilin anf freier Flamme erhitzt. Sobald die Flüssigkeit anfängt sich zu klären (bei etwa $150^{\circ}$ ), wird die Flamme rasch entfernt. Die Lösung geräth bald in heftiges Sieden, zugleich scheidet sich das Condensationsproduct in farblosen Blättchen $a b$, die nach dem Waschen mit Salzsäure und Wasser fast rein sind. In dem Dimethylanilin sind nur noch geringe Mengen des Körpers gelöst. Ausbente bis 60 pC. der Theorie. Der Körper bildet ans Wasser umkrystallisirt weisse Blättchen vom Schmelzp. $262^{\circ}$ (genau gleichzeitig mit einem zum Vergleiche nach Pinner dargestellten Präparate von Phenylurazol) und zeigt die schon beschriebenen Eigenschaften desselben.

1. $0,200 \overline{7} \mathrm{~g}$ gaben $0,4090 \mathrm{CO}_{\mathrm{a}}$, entsprechend $0,11154 \mathrm{C}$, u. 0,0760 $\mathrm{H}_{2} \mathrm{O}$, entsprechend $0,00844 \mathrm{H}$.

$0,1326 \mathrm{~g}$ gaben $28,2 \mathrm{~cm}$ Stickgas bci $11^{\circ}$ und $719 \mathrm{~mm}$ Druck.

II. $0,1385 \mathrm{~g}$ gaben $0,2751 \mathrm{CO}_{\mathrm{g}}$, entsprechend $0,07503 \mathrm{C}, \mathrm{u.} 0,0512 \mathrm{H}_{2} \mathrm{O}$, entsprechend $0,00568 \mathrm{H}$.

0,1140 g gaben 24,0 ecm Stickgas bei $9^{0}$ und $713 \mathrm{~mm}$ Druck.

\begin{tabular}{ccrr} 
& Berechnet für & \multicolumn{2}{c}{ Gefunden } \\
& $\mathrm{C}_{8} \mathrm{H}_{7} \mathrm{~N}_{3} \mathrm{O}_{2}$ & I. & II. \\
$\mathrm{C}$ & 54,23 & 54,22 & 54,17 \\
$\mathrm{H}$ & 3,95 & 4,10 & 4,10 \\
$\mathrm{C}$ & 23,72 & 23,97 & 23,73
\end{tabular}

Von alkoholischem Kali wird der Körper erst bei $250^{\circ}$ bis $280^{\circ}$ angegriffen, und liefert als Spaltungsproducte Kohlen-

14) Ber. d. deutsch. chem. Ges. 20, 2360. 
säure, Ammoniak und Phenylhydrazin, ein weiterer Beweis, dass Phenylurazol und nicht Phenyldioxyosotriazol vorliegt.

Mononatriumsalz. Entsteht beim Auflösen des Phenylurazols in der berechneten Menge Soda. Neutral reagirende Nadeln, leicht in Wasser, schwer in Alkohol löslich.

$0,2112 \mathrm{~g}$ gaben $0,0747 \mathrm{Na}_{2} \mathrm{SO}_{4}$.

Berechnet für Gefunden

$$
\mathrm{C}_{8} \mathrm{~N}_{8} \mathrm{H}_{4} \mathrm{O}_{2} \mathrm{Na}
$$

$\mathrm{Na} \quad 11,53$

11,46

Dinatriumsalz. $2 \mathrm{~g}$ Phenylurazol in $200 \mathrm{ccm}$ Alkohol gelöst, werden mit einer Lösung von zwei Molekülen Natriumäthylat versetzt. Beim Erhitzen zum Sieden scheidet sich das Dinatriumsalz als Pulver aus, welches anch beim Erkalten sich nur theilweise wieder auflöst. Siedend abfiltrirt und mit heissem Alkohol gewaschen, zeigt das Salz einen etwas zu geringen Natriumgehalt, offenbar in Folge einer geringen Zersetzung. Seine wässrige İösung reagirt stark alkalisch.

I. $0,3085 \mathrm{~g}$ gaben $0,1920 \mathrm{Na}_{2} \mathrm{SO}_{4}$.

II. $0,3094 \mathrm{~g} \quad, \quad 0,1920 \mathrm{Na}_{9} \mathrm{SO}_{4}$.

\begin{tabular}{|c|c|}
\hline Berechnet für & Gefunden \\
\hline $\mathrm{C}_{8} \mathrm{~N}_{3} \mathrm{H}_{5} \mathrm{O}_{2} \mathrm{Na}_{2}$ & II. \\
\hline 20,81 & 20,12 \\
\hline
\end{tabular}

Diacetylphenylurazol entsteht bei dreistündigem Kochen des Phenylurazols mit der fünffachen Menge Essigsäureanhydrid. Die I,ösung wird zur Trockne verdampft und der Rückstand aus trockncm Benzol umkrystallisirt. Weisse Nadeln. Schmelzp. 162-163\%.

$0,1783 \mathrm{~g}$ gaben $0,3627 \mathrm{CO}_{2}$, entsprechend $0,09891 \mathrm{C}$, und $0,0696 \mathrm{H}_{2} \mathrm{O}$, entsprechend $0,00773 \mathrm{H}$.

0,1595 g gaben 23,4 ecm Stickgas bei $11^{\circ}$ und 705, 5 mm Druck.

$\begin{array}{ccr} & \text { Berechnet für } & \text { Gefunder } \\ & \mathrm{C}_{8} \mathrm{H}_{5} \mathrm{~N}_{3} \mathrm{O}_{2}\left(\mathrm{CH}_{3} \mathrm{CO}\right)_{3} & \\ \mathrm{C} & 55,17 & 55,47 \\ \mathrm{H} & 4,21 & 4,34 \\ \mathrm{~N} & 16,09 & \mathbf{1 6 , 2 2}\end{array}$

Reaction auf feuchtem Lakmuspapier neutral. Pinner ${ }^{15}$ ) giebt für sein Diacetylurazol den Schmelzp. $141-149^{\circ}$ an,

15) Ber. d. deutsch. chem. Ges. 21, 1224. 
sodass es auf den ersten Blick schien, als ob der Körper aus Phenylhydrazidoxalhydroxamsäure doch verschieden wäre von dem Phenylurazol. Behandelt man Phenylurazol (nach Pinner dargestellt) indessen nach obiger Vorschrift, so erhält man dasselbe neutral reagirende Diacetylderivat vom Schmelzp. $162^{\circ}$.

$0,2480 \mathrm{~g}$ gaben $36,0 \mathrm{~cm}$ Stickgas bei $12^{\circ}$ und $711 \mathrm{~mm}$ Druck.

$$
\begin{array}{ccc} 
& \text { Berechnet für } & \text { Gefunden } \\
& \mathrm{C}_{8} \mathrm{H}_{5} \mathrm{~N}_{3} \mathrm{O}_{3}\left(\mathrm{COCHH}_{3}\right)_{2} & \\
\mathrm{~N} & 16,09 & 16,10
\end{array}
$$

Der von Pinner erhaltene Körper war offenbar ein Gemiseh von Mono- und Diacetylderivat. Kocht man Phenylurazol nach seiner Vorschrift mit der doppeiten Menge Anhydrid und verdunstet nach Zusatz von Alkohol, so erhält man durch Umkrystallisiren aus Benzol thatsächlich einen bei $140^{\circ}$ schmelzenden Körper, der auf feuchtes Lakmuspapier saner reagirt und einen zu hohen Stickstoffgehalt hat. Ein aus Monound Diacetylderivat bereitetes Gemisch schmolz ebenfalls bei $140-145^{\circ}$.

Monoacetylphenylurazol entsteht unter Abspaltung ron Essigsäure beim Kochen des Diacetylderivates mit Wasser. Man erhält es am besten durch kurzes Kochen des Phenylurazols mit Essigsäureanhydrid und Umkrystallisiren des Reactionsproductes aus Wasser. Der Körper krystallisirt aus Wasser oder Benzol in farblosen Nadeln vom Schmelzp. $170^{\circ}$, die auf feuchtes Lakmuspapier sauer reagiren.

$0,1971 \mathrm{~g}$ gaben $0,3958 \mathrm{CO}_{2}$, entsprechend $0,10794 \mathrm{C}$, und $0,0718 \mathrm{H}_{2} \mathrm{O}$, entsprechend $0,00796 \mathrm{H}$.

$0,2006 \mathrm{~g}$ gaben $34,3 \mathrm{ccm}$ Stickgas bei $8^{0}$ und $716 \mathrm{~mm}$ Druck.

$\begin{array}{ccr} & \text { Berechnet für } & \text { Gefunden } \\ & \mathrm{C}_{10} \mathrm{~N}_{3} \mathrm{H}_{9} \mathrm{O}_{3} & \\ \mathrm{C} & 54,79 & 54,76 \\ \mathrm{H} & 4,10 & 4,04 \\ \mathrm{~N} & 19,17 & 19,44\end{array}$

